Supporting Information for

\title{
Triple-Stranded Cluster Helicates for Selective Catalytic Oxidation of C-H bonds
}

\author{
Yu Fang, ${ }^{\dagger} \S$ Wei Gong ${ }^{\dagger, \S}$ Lujia Liu, ${ }^{\dagger}$ Yan Liu, ${ }^{* \dagger}$ and Yong Cui ${ }^{*}, \dagger$ \\ $\dagger$ School of Chemistry and Chemical Engineering and State Key Laboratory of Metal \\ Matrix Composites, Shanghai Jiao Tong University, Shanghai 200240, China \\ * Collaborative Innovation Center of Chemical Science and Engineering, Tianjin \\ 300072, China \\ E-mail: yongcui@sjtu.edu.cn, liuy@sjtu.edu.cn
}

\section{Table of Content}

1. Materials and general procedures

2. Synthesis of the ligand

3. Synthesis of $\mathbf{1}$ and $\mathbf{2}$

4. Table S1. Crystal data and structure refinement for $\mathbf{1}$ and $\mathbf{2}$

5. Tables S2-S3. Selected Bond lengths and angles for $\mathbf{1}$ and $\mathbf{2}$

6. Figures S1-S3. Additional X-ray crystallographic structures of $\mathbf{1}$ and $\mathbf{2}$

7. Figures S4-S6. UV/Vis absorption and fluorescent emission spectra

8. Experimental procedure of $\mathbf{1}$ catalyzed oxidation reactions

9. Tables S4. Peroxidative oxidation of toluene and ethyl benzene

10. GC and GC-MS results

11. GC results of recycle experiment

12. Oxidation of cyclohexane in the presence of radical trap

13. ${ }^{1} \mathrm{H}$ and ${ }^{13} \mathrm{C}$ NMR spectra

14. ESI-MS spectra 


\section{Materials and General Procedures}

All of the chemicals are commercial available, and used without further purification. Solvents for air sensitive reactions were distilled under nitrogen. Elemental analyses were performed with an EA1110 CHNS-0 CE elemental analyzer. The IR spectrum (KBr pellet) was recorded $\left(400-4000 \mathrm{~cm}^{-1}\right.$ region) on a Nicolet Magna 750 FT-IR spectrometer. Fluorescence measurements were carried out on a LS 50B Luminescence Spectrometer (Perkin Elmer, Inc., USA). UV/Vis absorption spectra were recorded on a Lambda $20 \mathrm{UV} /$ Vis Spectrometer (Perkin Elmer, Inc., USA). ${ }^{1} \mathrm{H}$ and ${ }^{13} \mathrm{C}$ NMR experiments were carried out on a MERCURYplus 400 spectrometer operating at resonance frequencies of $400 \mathrm{MHz}$. GC spectra were performed and recorded using a Lunan Ruihong SP-6890 equipped with a flame ionization detector and silica capillary column SP-56. GC-MS experiments were done by SHIMADZU GC-2010 with a coupled GCMS-QP2010, using GCMS Solution Version 2.5 (SHIMADZU Corp.). ESI-MS (Electrospray ionization mass spectra) were recorded on a Finnigan LCQ mass spectrometerusing $\mathrm{CH}_{2} \mathrm{CL}_{2}-\mathrm{MeOH}$ as mobile phase.

X-ray Crystallography. Single-crystal XRD data for compounds $\mathbf{1}$ and $\mathbf{2}$ were all collected on a Bruker SMART Apex II CCD-based X-ray diffractometer with $\mathrm{Cu}-\mathrm{Ka}$ radiation $(\lambda=1.54178 \AA$ ) at $296 \mathrm{~K}$. The empirical absorption correction was applied by using the SADABS program (G. M. Sheldrick, SADABS, program for empirical absorption correction of area detector data; University of Göttingen, Göttingen, Germany, 1996). The structure was solved using direct method, and refined by full-matrix least-squares on $\mathrm{F}^{2}$ (G. M. Sheldrick, SHELXTL97, program for crystal structure refinement, University of Göttingen, Germany, 1997). The hydrogen atoms were fixed at their calculated positions. All calculations were performed using APEX2 software for data collection and OLEX2-1.2 (J. Appl.Crystallogr. 2009, 42, 339-341.) and SHELXTL (Acta Crystallogr. Sect. A 2008, 64, 112-122; Sheldrick, G. M. SHELXT-2014, 2013) to resolve and refine the structure. In both 1 and 2, because of the relatively high anisotropic atomic displacement parameter, some of tert-butyl groups were satisfactorily modeled with the positional disorder and were treated with FVAR. All of the disordered parts and some other atoms were restrained using AFIX, FLAT, DFIX, ISOR, DELU, RIGU, and SIMU instructions to make the displacement parameters more reasonable. In compound $\mathbf{1}$, because of the relatively high isotropic atomic displacement parameter of two DMF molecules, these molecules were only refined isotropically without adding $\mathrm{H}$-atoms, while all other atoms were refined anisotropically. In compound $\mathbf{2}$, all atoms were refined anisotropically and added hydrogen atoms except one disordered DMF molecule. Crystal data and details of the 
data collection are given in Table S1, while the selected bond distances and angles are presented in Tables $\mathbf{S 2}$ and $\mathbf{S 3}$.

\section{Synthesis of the ligand}

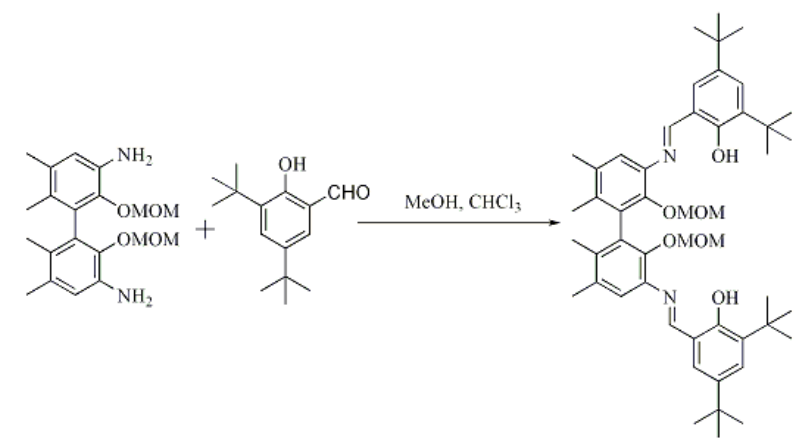

3,3'-diamino-5,5',6,6'-tetramethyl-2,2'-bis(methoxymethoxy)-1,1'-biphenyl was prepared according to the reported procedures (Angew. Chem. Int. Ed, 2011, 50, 1154). A mixture of 3,3'-diamino-5,5',6,6'-tetramethyl-2,2'-bis(methoxymethoxy)1,1'-biphenyl (0.75 g, $2.08 \mathrm{mmol})$ and 3,5-di-tert-butyl salicylaldehyde (4eq, $1.96 \mathrm{~g}$, $8.36 \mathrm{mmol})$ in mixture of $\mathrm{MeOH}$ and $\mathrm{CHCl}_{3}(\mathrm{v} / \mathrm{v}=1: 1,80 \mathrm{~mL})$ was stirred at r.t. for 12 $\mathrm{h}$, and the mixture was then filtered to collect the solid product, which could be purified by washed with cold hexane $(0.57 \mathrm{~g}, 77 \%)$.

${ }^{1} \mathrm{H} \quad \mathrm{NMR} \quad\left(\mathrm{CDCl}_{3}, 400 \mathrm{MHz}\right): 13.757(\mathrm{~s}, \quad 2 \mathrm{H}, \mathrm{OH}), 8.718(\mathrm{~s}, \quad 2 \mathrm{H}, \mathrm{C}=\mathrm{NH})$, 7.432-7.427(d, $J=2.0 \mathrm{~Hz}, 2 \mathrm{H}, \operatorname{ArH}), 7.244-7.237(\mathrm{~d}, J=2.8 \mathrm{~Hz}, 2 \mathrm{H}, \operatorname{ArH}), 7.052(\mathrm{~s}$, 2H, ArH), 4.883-4.807(dd, $\left.J=15.2 \mathrm{~Hz}, 4 \mathrm{H}, \mathrm{CH}_{2}\right), 2.859(\mathrm{~s}, 6 \mathrm{H}, \mathrm{Me}), 2.340(\mathrm{~s}, 6 \mathrm{H}$, $\mathrm{Me}), 1.989(\mathrm{~s}, 6 \mathrm{H}, \mathrm{Me}), 1.448\left(\mathrm{~s}, 18 \mathrm{H}\right.$, tert-butyl), $1.335\left(\mathrm{~s}, 18 \mathrm{H}\right.$, tert-butyl); ${ }^{13} \mathrm{C}$ NMR $\left(\mathrm{CDCl}_{3}, 400 \mathrm{MHz}\right): 163.884,158.503,147.244,140.501,139.399,137.172,135.592$, $133.300,133.257,128.031,126.900,120.324,118.661,99.527,77.552,77.235$, 76.920, 56.357, 35.314, 34.405, 31.720, 29.584, 20.514, 17.149. ESI-MS: m/z 705.6 (Calcd m/z 705.5 for $\left.[\mathrm{L}+\mathrm{H}]^{+}\right)$.

\section{Synthesis of 1 and 2}

A mixture of $\mathrm{CuSO}_{4} \cdot 5 \mathrm{H}_{2} \mathrm{O}$ or $\mathrm{Zn}(\mathrm{OAc})_{2} \cdot 2 \mathrm{H}_{2} \mathrm{O}(0.1 \mathrm{mmol})$, L-(2MOM-2H) $(0.05$ mmol) in $\mathrm{H}_{2} \mathrm{O}(0.5 \mathrm{~mL})$, DMF $(0.75 \mathrm{~mL}), \mathrm{CHCl}_{3}(0.2 \mathrm{~mL})$ and $\mathrm{CH}_{3} \mathrm{CN}(0.1 \mathrm{~mL})$ was stirred for 10 min to give a cloudy solution and then it was allowed to stand at $100^{\circ} \mathrm{C}$. After two days, blue block or orange cubic crystals suitable for single-crystal X-ray diffraction were collected, washed with ether and dried in air $(20.7 \mathrm{mg}, 81 \%$ for $\mathbf{1}$ and $22.7 \mathrm{mg}, 85 \%$ for 2 ).

Anal (\%).Calcd for $1\left[\mathrm{Cu}_{7}(\mathrm{OH})_{2} \mathbf{L}_{3}\right] \cdot 2 \mathrm{DMF} \cdot 2 \mathrm{H}_{2} \mathrm{O} / \mathrm{C}_{144} \mathrm{H}_{188} \mathrm{Cu}_{7} \mathrm{~N}_{8} \mathrm{O}_{18}: \mathrm{C}, 62.58 ; \mathrm{H}$, 6.86; N, 4.05. Found: C, 62.22; H, 6.84; N, 4.02. FTIR (KBr pellet): 3445.70(m), 2935.12(m), 2364.83(w), 1608.30(s), 1540.29(w), 1463.79(m), 1384.46(s), 1322.73(s), 
1299.03(s), 1218.31(s), 1049.30(s), 957.98(w), 836.10(m), 777.15(w), 701.81(w), 652.65(w).

Anal (\%).Calcd for $2\left[\mathrm{Zn}_{7}(\mathrm{OH})_{2} \mathbf{L}_{3}\right] \cdot 6 \mathrm{DMF} \cdot 2 \mathrm{H}_{2} \mathrm{O} / \mathrm{C}_{156} \mathrm{H}_{216} \mathrm{Zn}_{7} \mathrm{~N}_{12} \mathrm{O}_{22}: \mathrm{C}, 61.05 ; \mathrm{H}$, 7.09; N, 5.48;. Found: C, 61.12; H, 7.03; N, 5.45. FTIR (KBr pellet): 3489.90(s), 2932.50(s), 2461.53(w), 1606.81(s), 1539.72(m), 1506.05(w), 1456.22(m), 1423.89(s), 1388.49(s), 1325.55(w), 1222.29(m), 1059.26(s), 835.20(m), 721.57(m), 568.06(w). The guest molecules can be readily removed by heating in vacuo for ${ }^{1} \mathrm{H}$ and ${ }^{13} \mathrm{C}$ NMR. ${ }^{1} \mathrm{H}$ NMR $\left(\mathrm{CDCl}_{3}, 400 \mathrm{MHz}\right): 8.389(\mathrm{~s}, 2 \mathrm{H}, \mathrm{C}=\mathrm{NH}), 7.236-7.242(\mathrm{~d}, J=2.4 \mathrm{~Hz}, 2 \mathrm{H}$, ArH), 7.104(s, 2H, ArH), 6.960-6.966(d, $J=2.4 \mathrm{~Hz}, 2 \mathrm{H}, \mathrm{ArH}), 2.034(\mathrm{~s}, 6 \mathrm{H}, \mathrm{Me})$, $1.326(\mathrm{~s}, 18 \mathrm{H}$, tert-butyl), 0.963(s, $18 \mathrm{H}$, tert-butyl), 0.742(s, 6H, Me). 


\section{Table S1. Crystal data and structure refinement for 1 and 2}

\begin{tabular}{|c|c|c|}
\hline Identification code & 1 & 2 \\
\hline CCDC number & 763149 & 763150 \\
\hline Empirical formula & $\mathrm{C}_{144} \mathrm{H}_{168} \mathrm{Cu}_{7} \mathrm{~N}_{8} \mathrm{O}_{16}$ & $\mathrm{C}_{159} \mathrm{H}_{210} \mathrm{Zn}_{7} \mathrm{~N}_{13} \mathrm{O}_{21}$ \\
\hline Formula weight & 2711.63 & 3096.98 \\
\hline Temperature $(\mathrm{K})$ & $296(2)$ & $296(2)$ \\
\hline Wavelength $(\AA)$ & 1.54178 & 1.54178 \\
\hline Crystal system & Triclinic & Monoclinic \\
\hline Space group & $\mathrm{P}-1$ & $\mathrm{C} 2 / \mathrm{c}$ \\
\hline Unit cell dimensions & $\begin{array}{l}\mathrm{a}=17.4508(6) \AA \\
\mathrm{b}=18.7149(6) \AA \\
\mathrm{c}=23.8379(8) \AA \\
\mathrm{alpha}=97.4850^{\circ} \\
\text { beta }=94.706(2)^{\mathrm{o}} \\
\text { gamma }=98.437(2)^{\mathrm{o}}\end{array}$ & $\begin{array}{l}\mathrm{a}=20.7405(4) \AA \\
\mathrm{b}=24.2320(5) \AA \\
\mathrm{c}=32.6921(5) \AA \\
\text { alpha }=90^{\circ} \\
\text { beta }=97.999^{\circ} \\
\text { gamma }=90^{\circ}\end{array}$ \\
\hline Volume $\left(\AA^{3}\right), Z$ & 7594.6(4), 2 & $16270.7(5), 4$ \\
\hline Density (calculated) $\left(\mathrm{mg} / \mathrm{m}^{3}\right)$ & 1.186 & 1.264 \\
\hline Absorption coefficient $\left(\mathrm{mm}^{-1}\right)$ & 1.517 & 1.649 \\
\hline $\mathrm{F}(000)$ & 2838 & 6532 \\
\hline$\theta$ range for data collection $\left({ }^{\circ}\right)$ & 2.412 to 50.00 & 2.730 to 54.997 \\
\hline Limiting indices & $\begin{array}{l}-17 \leq \mathrm{h} \leq 17,-17 \leq \mathrm{k} \leq 18,-23 \leq \mathrm{I} \\
\leq 23\end{array}$ & $\begin{array}{l}-22 \leq \mathrm{h} \leq 21,-17 \leq \mathrm{k} \leq 25,-34 \leq \\
\mathrm{l} \leq 34\end{array}$ \\
\hline Reflections collected & 22142 & 22832 \\
\hline Independent reflections & $14157[\mathrm{R}($ int $)=0.0365]$ & $9551[\mathrm{R}($ int $)=0.0234]$ \\
\hline Completeness to theta & $50.00 / 90.6 \%$ & $54.997 / 93.6 \%$ \\
\hline Refinement method & Full-matrix least-squares on $\mathrm{F}^{2}$ & Full-matrix least-squares on $\mathrm{F}^{2}$ \\
\hline Data / restraints / parameters & $14157 / 219 / 1449$ & $9551 / 349 / 947$ \\
\hline Goodness-of-fit on $\mathrm{F}^{\wedge} 2$ & 1.040 & 1.084 \\
\hline Final $R$ indices $[\mathrm{I}>2 \operatorname{sigma}(\mathrm{I})]$ & $\mathrm{R} 1=0.0690, \mathrm{wR} 2=0.2000$ & $\mathrm{R} 1=0.0558, \mathrm{wR} 2=0.1536$ \\
\hline $\mathrm{R}$ indices (all data) & $\mathrm{R} 1=0.0822, \mathrm{wR} 2=0.2150$ & $\mathrm{R} 1=0.0652, \mathrm{wR} 2=0.1685$ \\
\hline Largest diff. peak and hole $\left(\mathrm{e} . \AA^{-3}\right)$ & 0.626 and -0.451 & 1.154 and -0.511 \\
\hline
\end{tabular}




\section{Selected Bond lengths and angles for 1 and 2}

5.1. Table S2. Selected bond lengths $[\AA]$ and angles $\left[{ }^{\circ}\right]$ for $\mathbf{1}$.

\begin{tabular}{|c|c|}
\hline $\mathrm{Cu}(1)-\mathrm{O}(1)$ & $2.170(5)$ \\
\hline $\mathrm{Cu}(1)-\mathrm{O}(3)$ & $2.160(5)$ \\
\hline $\mathrm{Cu}(1)-\mathrm{O}(5)$ & $2.139(5)$ \\
\hline $\mathrm{Cu}(1)-\mathrm{O}(7)$ & $2.047(5)$ \\
\hline $\mathrm{Cu}(1)-\mathrm{O}(9)$ & $2.052(5)$ \\
\hline $\mathrm{Cu}(1)-\mathrm{O}(11)$ & $2.162(5)$ \\
\hline $\mathrm{Cu}(2)-\mathrm{O}(3)$ & $2.433(5)$ \\
\hline $\mathrm{Cu}(2)-\mathrm{O}(7)$ & $1.976(4)$ \\
\hline $\mathrm{Cu}(2)-\mathrm{O}(8)$ & $1.865(5)$ \\
\hline $\mathrm{Cu}(2)-\mathrm{O}(13)$ & $1.996(4)$ \\
\hline $\mathrm{Cu}(2)-\mathrm{N}(4)$ & $1.904(6)$ \\
\hline $\mathrm{Cu}(3)-\mathrm{O}(3)$ & $1.964(4)$ \\
\hline $\mathrm{Cu}(3)-\mathrm{O}(4)$ & $1.856(5)$ \\
\hline $\mathrm{Cu}(3)-\mathrm{O}(13)$ & $1.974(5)$ \\
\hline $\mathrm{Cu}(3)-\mathrm{N}(2)$ & $1.912(6)$ \\
\hline $\mathrm{Cu}(4)-\mathrm{O}(11)$ & $1.961(5)$ \\
\hline $\mathrm{Cu}(4)-\mathrm{O}(12)$ & $1.868(5)$ \\
\hline $\mathrm{Cu}(4)-\mathrm{O}(13)$ & $1.990(4)$ \\
\hline $\mathrm{Cu}(4)-\mathrm{N}(6)$ & $1.893(6)$ \\
\hline $\mathrm{Cu}(5)-\mathrm{O}(9)$ & $1.966(4)$ \\
\hline $\mathrm{Cu}(5)-\mathrm{O}(10)$ & $1.856(5)$ \\
\hline $\mathrm{Cu}(5)-\mathrm{O}(14)$ & $2.000(4)$ \\
\hline $\mathrm{Cu}(5)-\mathrm{N}(5)$ & $1.907(6)$ \\
\hline $\mathrm{Cu}(6)-\mathrm{O}(5)$ & $1.950(5)$ \\
\hline $\mathrm{Cu}(6)-\mathrm{O}(6)$ & $1.850(6)$ \\
\hline $\mathrm{Cu}(6)-\mathrm{O}(14)$ & $1.970(4)$ \\
\hline $\mathrm{Cu}(6)-\mathrm{N}(3)$ & $1.905(6)$ \\
\hline $\mathrm{Cu}(7)-\mathrm{O}(1)$ & $1.966(4)$ \\
\hline $\mathrm{Cu}(7)-\mathrm{O}(2)$ & $1.880(5)$ \\
\hline $\mathrm{Cu}(7)-\mathrm{O}(14)$ & $1.982(5)$ \\
\hline $\mathrm{Cu}(7)-\mathrm{N}(1)$ & $1.904(6)$ \\
\hline $\mathrm{O}(3)-\mathrm{Cu}(1)-\mathrm{O}(1)$ & $87.27(16)$ \\
\hline $\mathrm{O}(3)-\mathrm{Cu}(1)-\mathrm{O}(11)$ & $83.15(17)$ \\
\hline $\mathrm{O}(5)-\mathrm{Cu}(1)-\mathrm{O}(1)$ & $85.37(17)$ \\
\hline $\mathrm{O}(5)-\mathrm{Cu}(1)-\mathrm{O}(3)$ & $170.08(17)$ \\
\hline $\mathrm{O}(5)-\mathrm{Cu}(1)-\mathrm{O}(11)$ & $104.87(17)$ \\
\hline $\mathrm{O}(7)-\mathrm{Cu}(1)-\mathrm{O}(1)$ & $102.06(18)$ \\
\hline $\mathrm{O}(7)-\mathrm{Cu}(1)-\mathrm{O}(3)$ & $85.06(17)$ \\
\hline $\mathrm{O}(7)-\mathrm{Cu}(1)-\mathrm{O}(5)$ & $89.99(17)$ \\
\hline $\mathrm{O}(7)-\mathrm{Cu}(1)-\mathrm{O}(9)$ & $171.56(17)$ \\
\hline
\end{tabular}




\begin{tabular}{|c|c|}
\hline $\mathrm{O}(7)-\mathrm{Cu}(1)-\mathrm{O}(11)$ & $83.71(17)$ \\
\hline $\mathrm{O}(9)-\mathrm{Cu}(1)-\mathrm{O}(1)$ & $84.10(17)$ \\
\hline $\mathrm{O}(9)-\mathrm{Cu}(1)-\mathrm{O}(3)$ & $101.12(18)$ \\
\hline $\mathrm{O}(9)-\mathrm{Cu}(1)-\mathrm{O}(5)$ & $84.73(18)$ \\
\hline $\mathrm{O}(9)-\mathrm{Cu}(1)-\mathrm{O}(11)$ & $91.28(17)$ \\
\hline $\mathrm{O}(11)-\mathrm{Cu}(1)-\mathrm{O}(1)$ & $168.38(17)$ \\
\hline $\mathrm{O}(7)-\mathrm{Cu}(2)-\mathrm{O}(3)$ & $79.60(17)$ \\
\hline $\mathrm{O}(7)-\mathrm{Cu}(2)-\mathrm{O}(13)$ & $87.52(18)$ \\
\hline $\mathrm{O}(8)-\mathrm{Cu}(2)-\mathrm{O}(3)$ & $98.2(2)$ \\
\hline $\mathrm{O}(8)-\mathrm{Cu}(2)-\mathrm{O}(7)$ & $177.8(2)$ \\
\hline $\mathrm{O}(8)-\mathrm{Cu}(2)-\mathrm{O}(13)$ & $92.0(2)$ \\
\hline $\mathrm{O}(8)-\mathrm{Cu}(2)-\mathrm{N}(4)$ & $96.1(2)$ \\
\hline $\mathrm{O}(13)-\mathrm{Cu}(2)-\mathrm{O}(3)$ & $74.48(17)$ \\
\hline $\mathrm{N}(4)-\mathrm{Cu}(2)-\mathrm{O}(3)$ & $126.6(2)$ \\
\hline $\mathrm{N}(4)-\mathrm{Cu}(2)-\mathrm{O}(7)$ & $85.2(2)$ \\
\hline $\mathrm{N}(4)-\mathrm{Cu}(2)-\mathrm{O}(13)$ & $155.6(2)$ \\
\hline $\mathrm{O}(3)-\mathrm{Cu}(3)-\mathrm{O}(13)$ & $86.69(18)$ \\
\hline $\mathrm{O}(4)-\mathrm{Cu}(3)-\mathrm{O}(3)$ & $178.1(2)$ \\
\hline $\mathrm{O}(4)-\mathrm{Cu}(3)-\mathrm{O}(13)$ & $93.2(2)$ \\
\hline $\mathrm{O}(4)-\mathrm{Cu}(3)-\mathrm{N}(2)$ & $95.4(2)$ \\
\hline $\mathrm{N}(2)-\mathrm{Cu}(3)-\mathrm{O}(3)$ & $85.3(2)$ \\
\hline $\mathrm{N}(2)-\mathrm{Cu}(3)-\mathrm{O}(13)$ & $160.0(2)$ \\
\hline $\mathrm{O}(11)-\mathrm{Cu}(4)-\mathrm{O}(13)$ & $87.50(19)$ \\
\hline $\mathrm{O}(12)-\mathrm{Cu}(4)-\mathrm{O}(11)$ & $176.4(2)$ \\
\hline $\mathrm{O}(12)-\mathrm{Cu}(4)-\mathrm{O}(13)$ & $91.8(2)$ \\
\hline $\mathrm{O}(12)-\mathrm{Cu}(4)-\mathrm{N}(6)$ & $95.7(3)$ \\
\hline $\mathrm{N}(6)-\mathrm{Cu}(4)-\mathrm{O}(11)$ & $86.1(2)$ \\
\hline $\mathrm{N}(6)-\mathrm{Cu}(4)-\mathrm{O}(13)$ & $160.3(2)$ \\
\hline $\mathrm{O}(9)-\mathrm{Cu}(5)-\mathrm{O}(14)$ & $89.10(18)$ \\
\hline $\mathrm{O}(10)-\mathrm{Cu}(5)-\mathrm{O}(9)$ & 179.1(2) \\
\hline $\mathrm{O}(10)-\mathrm{Cu}(5)-\mathrm{O}(14)$ & $90.4(2)$ \\
\hline $\mathrm{O}(10)-\mathrm{Cu}(5)-\mathrm{N}(5)$ & $95.5(2)$ \\
\hline $\mathrm{N}(5)-\mathrm{Cu}(5)-\mathrm{O}(9)$ & $85.2(2)$ \\
\hline $\mathrm{N}(5)-\mathrm{Cu}(5)-\mathrm{O}(14)$ & $158.4(2)$ \\
\hline $\mathrm{O}(5)-\mathrm{Cu}(6)-\mathrm{O}(14)$ & $87.42(19)$ \\
\hline $\mathrm{O}(6)-\mathrm{Cu}(6)-\mathrm{O}(5)$ & $175.3(3)$ \\
\hline $\mathrm{O}(6)-\mathrm{Cu}(6)-\mathrm{O}(14)$ & $92.2(2)$ \\
\hline $\mathrm{O}(6)-\mathrm{Cu}(6)-\mathrm{N}(3)$ & $96.3(3)$ \\
\hline $\mathrm{N}(3)-\mathrm{Cu}(6)-\mathrm{O}(5)$ & $85.5(2)$ \\
\hline $\mathrm{N}(3)-\mathrm{Cu}(6)-\mathrm{O}(14)$ & $160.5(2)$ \\
\hline $\mathrm{O}(1)-\mathrm{Cu}(7)-\mathrm{O}(14)$ & $86.84(19)$ \\
\hline $\mathrm{O}(2)-\mathrm{Cu}(7)-\mathrm{O}(1)$ & $177.6(2)$ \\
\hline $\mathrm{O}(2)-\mathrm{Cu}(7)-\mathrm{O}(14)$ & $93.5(2)$ \\
\hline $\mathrm{O}(2)-\mathrm{Cu}(7)-\mathrm{N}(1)$ & $94.9(3)$ \\
\hline
\end{tabular}




\begin{tabular}{|c|c|}
\hline $\mathrm{N}(1)-\mathrm{Cu}(7)-\mathrm{O}(1)$ & $85.5(2)$ \\
\hline $\mathrm{N}(1)-\mathrm{Cu}(7)-\mathrm{O}(14)$ & $159.7(2)$ \\
\hline $\mathrm{Cu}(7)-\mathrm{O}(1)-\mathrm{Cu}(1)$ & $105.3(2)$ \\
\hline $\mathrm{C}(17)-\mathrm{O}(1)-\mathrm{Cu}(1)$ & $122.3(4)$ \\
\hline $\mathrm{C}(17)-\mathrm{O}(1)-\mathrm{Cu}(7)$ & $109.3(3)$ \\
\hline $\mathrm{C}(1)-\mathrm{O}(2)-\mathrm{Cu}(7)$ & $127.4(5)$ \\
\hline $\mathrm{Cu}(1)-\mathrm{O}(3)-\mathrm{Cu}(2)$ & $88.75(16)$ \\
\hline $\mathrm{Cu}(3)-\mathrm{O}(3)-\mathrm{Cu}(1)$ & $106.0(2)$ \\
\hline $\mathrm{Cu}(3)-\mathrm{O}(3)-\mathrm{Cu}(2)$ & $91.04(18)$ \\
\hline $\mathrm{C}(25)-\mathrm{O}(3)-\mathrm{Cu}(1)$ & $122.2(4)$ \\
\hline $\mathrm{C}(25)-\mathrm{O}(3)-\mathrm{Cu}(2)$ & $133.9(3)$ \\
\hline $\mathrm{C}(25)-\mathrm{O}(3)-\mathrm{Cu}(3)$ & 109.1(3) \\
\hline $\mathrm{C}(34)-\mathrm{O}(4)-\mathrm{Cu}(3)$ & $127.5(4)$ \\
\hline $\mathrm{Cu}(6)-\mathrm{O}(5)-\mathrm{Cu}(1)$ & $106.9(2)$ \\
\hline $\mathrm{C}(63)-\mathrm{O}(5)-\mathrm{Cu}(1)$ & $121.2(4)$ \\
\hline $\mathrm{C}(63)-\mathrm{O}(5)-\mathrm{Cu}(6)$ & $108.6(3)$ \\
\hline $\mathrm{C}(47)-\mathrm{O}(6)-\mathrm{Cu}(6)$ & $127.8(4)$ \\
\hline $\mathrm{Cu}(2)-\mathrm{O}(7)-\mathrm{Cu}(1)$ & $106.24(19)$ \\
\hline $\mathrm{C}(71)-\mathrm{O}(7)-\mathrm{Cu}(1)$ & $123.2(3)$ \\
\hline $\mathrm{C}(71)-\mathrm{O}(7)-\mathrm{Cu}(2)$ & $108.7(3)$ \\
\hline $\mathrm{C}(80)-\mathrm{O}(8)-\mathrm{Cu}(2)$ & $127.1(4)$ \\
\hline $\mathrm{Cu}(5)-\mathrm{O}(9)-\mathrm{Cu}(1)$ & $107.9(2)$ \\
\hline $\mathrm{C}(109)-\mathrm{O}(9)-\mathrm{Cu}(1)$ & $124.4(4)$ \\
\hline $\mathrm{C}(109)-\mathrm{O}(9)-\mathrm{Cu}(5)$ & $109.2(3)$ \\
\hline $\mathrm{C}(93)-\mathrm{O}(10)-\mathrm{Cu}(5)$ & $127.3(4)$ \\
\hline $\mathrm{Cu}(4)-\mathrm{O}(11)-\mathrm{Cu}(1)$ & $106.4(2)$ \\
\hline $\mathrm{C}(117)-\mathrm{O}(11)-\mathrm{Cu}(1)$ & $120.4(4)$ \\
\hline $\mathrm{C}(117)-\mathrm{O}(11)-\mathrm{Cu}(4)$ & $109.3(3)$ \\
\hline $\mathrm{C}(126)-\mathrm{O}(12)-\mathrm{Cu}(4)$ & $126.2(4)$ \\
\hline $\mathrm{Cu}(3)-\mathrm{O}(13)-\mathrm{Cu}(2)$ & $105.2(2)$ \\
\hline $\mathrm{Cu}(3)-\mathrm{O}(13)-\mathrm{Cu}(4)$ & $103.8(2)$ \\
\hline $\mathrm{Cu}(4)-\mathrm{O}(13)-\mathrm{Cu}(2)$ & $105.02(19)$ \\
\hline $\mathrm{Cu}(6)-\mathrm{O}(14)-\mathrm{Cu}(5)$ & $103.4(2)$ \\
\hline $\mathrm{Cu}(6)-\mathrm{O}(14)-\mathrm{Cu}(7)$ & $107.3(2)$ \\
\hline $\mathrm{Cu}(7)-\mathrm{O}(14)-\mathrm{Cu}(5)$ & $105.0(2)$ \\
\hline $\mathrm{C}(15)-\mathrm{N}(1)-\mathrm{Cu}(7)$ & $124.7(5)$ \\
\hline $\mathrm{C}(15)-\mathrm{N}(1)-\mathrm{C}(16)$ & $124.2(6)$ \\
\hline $\mathrm{C}(16)-\mathrm{N}(1)-\mathrm{Cu}(7)$ & $111.0(4)$ \\
\hline $\mathrm{C}(26)-\mathrm{N}(2)-\mathrm{Cu}(3)$ & $111.0(4)$ \\
\hline $\mathrm{C}(32)-\mathrm{N}(2)-\mathrm{Cu}(3)$ & $123.4(5)$ \\
\hline $\mathrm{C}(32)-\mathrm{N}(2)-\mathrm{C}(26)$ & $125.6(6)$ \\
\hline $\mathrm{C}(61)-\mathrm{N}(3)-\mathrm{Cu}(6)$ & $123.0(5)$ \\
\hline $\mathrm{C}(61)-\mathrm{N}(3)-\mathrm{C}(62)$ & $124.9(6)$ \\
\hline $\mathrm{C}(62)-\mathrm{N}(3)-\mathrm{Cu}(6)$ & $111.9(4)$ \\
\hline
\end{tabular}




$\begin{array}{ll}\mathrm{C}(72)-\mathrm{N}(4)-\mathrm{Cu}(2) & 112.2(4) \\ \mathrm{C}(78)-\mathrm{N}(4)-\mathrm{Cu}(2) & 123.3(5) \\ \mathrm{C}(78)-\mathrm{N}(4)-\mathrm{C}(72) & 124.3(5) \\ \mathrm{C}(107)-\mathrm{N}(5)-\mathrm{Cu}(5) & 123.4(5) \\ \mathrm{C}(107)-\mathrm{N}(5)-\mathrm{C}(108) & 125.1(5) \\ \mathrm{C}(108)-\mathrm{N}(5)-\mathrm{Cu}(5) & 111.5(4) \\ \mathrm{C}(118)-\mathrm{N}(6)-\mathrm{Cu}(4) & 111.1(4) \\ \mathrm{C}(124)-\mathrm{N}(6)-\mathrm{Cu}(4) & 124.0(6)\end{array}$

5.2. Table S3. Selected bond lengths $[\AA]$ and angles $\left[{ }^{\circ}\right]$ for 2 .

\begin{tabular}{lc}
\hline $\mathrm{Zn}(1)-\mathrm{O}(2) \# 1$ & $2.113(3)$ \\
$\mathrm{Zn}(1)-\mathrm{O}(2)$ & $2.113(3)$ \\
$\mathrm{Zn}(1)-\mathrm{O}(3)$ & $2.075(3)$ \\
$\mathrm{Zn}(1)-\mathrm{O}(3) \# 1$ & $2.075(3)$ \\
$\mathrm{Zn}(1)-\mathrm{O}(6)$ & $2.108(3)$ \\
$\mathrm{Zn}(1)-\mathrm{O}(6) \# 1$ & $2.108(3)$ \\
$\mathrm{Zn}(2)-\mathrm{Zn}(3)$ & $3.1468(8)$ \\
$\mathrm{Zn}(2)-\mathrm{Zn}(4)$ & $3.1233(9)$ \\
$\mathrm{Zn}(2)-\mathrm{O}(2)$ & $2.021(3)$ \\
$\mathrm{Zn}(2)-\mathrm{O}(5)$ & $1.931(4)$ \\
$\mathrm{Zn}(2)-\mathrm{O}(6)$ & $2.306(3)$ \\
$\mathrm{Zn}(2)-\mathrm{O}(7)$ & $2.024(3)$ \\
$\mathrm{Zn}(2)-\mathrm{N}(3)$ & $1.993(4)$ \\
$\mathrm{Zn}(3)-\mathrm{Zn}(4)$ & $3.1102(9)$ \\
$\mathrm{Zn}(3)-\mathrm{O}(3) \# 1$ & $2.327(3)$ \\
$\mathrm{Zn}(3)-\mathrm{O}(4) \# 1$ & $1.933(3)$ \\
$\mathrm{Zn}(3)-\mathrm{O}(6)$ & $2.041(3)$ \\
$\mathrm{Zn}(3)-\mathrm{O}(7)$ & $2.027(3)$ \\
$\mathrm{Zn}(3)-\mathrm{N}(2) \# 1$ & $1.997(4)$ \\
$\mathrm{Zn}(4)-\mathrm{O}(1)$ & $1.933(3)$ \\
$\mathrm{Zn}(4)-\mathrm{O}(2)$ & $2.333(3)$ \\
$\mathrm{Zn}(4)-\mathrm{O}(3) \# 1$ & $2.004(3)$ \\
$\mathrm{Zn}(4)-\mathrm{O}(7)$ & $2.043(3)$ \\
$\mathrm{Zn}(4)-\mathrm{N}(1)$ & $1.977(4)$ \\
$\mathrm{O}(3)-\mathrm{Zn}(3) \# 1$ & $2.327(3)$ \\
$\mathrm{O}(3)-\mathrm{Zn}(4) \# 1$ & $2.004(3)$ \\
$\mathrm{O}(4)-\mathrm{Zn}(3) \# 1$ & $1.933(3)$ \\
$\mathrm{N}(2)-\mathrm{Zn}(3) \# 1$ & $1.997(4)$ \\
& \\
$\mathrm{O}(2) \# 1-\mathrm{Zn}(1)-\mathrm{O}(2)$ & \\
$\mathrm{O}(3) \# 1-\mathrm{Zn}(1)-\mathrm{O}(2)$ & \\
& \\
& \\
& \\
& \\
& \\
& \\
&
\end{tabular}




\begin{tabular}{|c|c|}
\hline $\mathrm{O}(3)-\mathrm{Zn}(1)-\mathrm{O}(2) \# 1$ & $82.18(11)$ \\
\hline $\mathrm{O}(3)-\mathrm{Zn}(1)-\mathrm{O}(2)$ & $88.29(11)$ \\
\hline $\mathrm{O}(3) \# 1-\mathrm{Zn}(1)-\mathrm{O}(2) \# 1$ & $88.29(11)$ \\
\hline $\mathrm{O}(3)-\mathrm{Zn}(1)-\mathrm{O}(3) \# 1$ & $163.08(17)$ \\
\hline $\mathrm{O}(3) \# 1-\mathrm{Zn}(1)-\mathrm{O}(6)$ & $83.38(11)$ \\
\hline $\mathrm{O}(3)-\mathrm{Zn}(1)-\mathrm{O}(6) \# 1$ & $83.38(11)$ \\
\hline $\mathrm{O}(3)-\mathrm{Zn}(1)-\mathrm{O}(6)$ & $109.35(12)$ \\
\hline $\mathrm{O}(3) \# 1-\mathrm{Zn}(1)-\mathrm{O}(6) \# 1$ & $109.35(12)$ \\
\hline $\mathrm{O}(6) \# 1-\mathrm{Zn}(1)-\mathrm{O}(2)$ & $162.53(12)$ \\
\hline $\mathrm{O}(6)-\mathrm{Zn}(1)-\mathrm{O}(2) \# 1$ & $162.53(12)$ \\
\hline $\mathrm{O}(6)-\mathrm{Zn}(1)-\mathrm{O}(2)$ & $82.78(12)$ \\
\hline $\mathrm{O}(6) \# 1-\mathrm{Zn}(1)-\mathrm{O}(2) \# 1$ & $82.77(12)$ \\
\hline $\mathrm{O}(6)-\mathrm{Zn}(1)-\mathrm{O}(6) \# 1$ & $85.53(15)$ \\
\hline $\mathrm{Zn}(4)-\mathrm{Zn}(2)-\mathrm{Zn}(3)$ & $59.48(2)$ \\
\hline $\mathrm{O}(2)-\mathrm{Zn}(2)-\mathrm{Zn}(3)$ & $87.80(9)$ \\
\hline $\mathrm{O}(2)-\mathrm{Zn}(2)-\mathrm{Zn}(4)$ & $48.30(8)$ \\
\hline $\mathrm{O}(2)-\mathrm{Zn}(2)-\mathrm{O}(6)$ & $80.03(12)$ \\
\hline $\mathrm{O}(2)-\mathrm{Zn}(2)-\mathrm{O}(7)$ & $87.22(12)$ \\
\hline $\mathrm{O}(5)-\mathrm{Zn}(2)-\mathrm{Zn}(3)$ & $143.52(12)$ \\
\hline $\mathrm{O}(5)-\mathrm{Zn}(2)-\mathrm{Zn}(4)$ & $105.54(13)$ \\
\hline $\mathrm{O}(5)-\mathrm{Zn}(2)-\mathrm{O}(2)$ & $106.64(16)$ \\
\hline $\mathrm{O}(5)-\mathrm{Zn}(2)-\mathrm{O}(6)$ & $171.24(15)$ \\
\hline $\mathrm{O}(5)-\mathrm{Zn}(2)-\mathrm{O}(7)$ & $106.90(15)$ \\
\hline $\mathrm{O}(5)-\mathrm{Zn}(2)-\mathrm{N}(3)$ & $94.59(16)$ \\
\hline $\mathrm{O}(6)-\mathrm{Zn}(2)-\mathrm{Zn}(3)$ & $40.39(7)$ \\
\hline $\mathrm{O}(6)-\mathrm{Zn}(2)-\mathrm{Zn}(4)$ & $83.11(7)$ \\
\hline $\mathrm{O}(7)-\mathrm{Zn}(2)-\mathrm{Zn}(3)$ & $39.08(9)$ \\
\hline $\mathrm{O}(7)-\mathrm{Zn}(2)-\mathrm{Zn}(4)$ & $40.07(9)$ \\
\hline $\mathrm{O}(7)-\mathrm{Zn}(2)-\mathrm{O}(6)$ & $78.71(11)$ \\
\hline $\mathrm{N}(3)-\operatorname{Zn}(2)-\operatorname{Zn}(3)$ & $105.01(11)$ \\
\hline $\mathrm{N}(3)-\mathrm{Zn}(2)-\mathrm{Zn}(4)$ & $159.73(11)$ \\
\hline $\mathrm{N}(3)-\mathrm{Zn}(2)-\mathrm{O}(2)$ & $123.71(14)$ \\
\hline $\mathrm{N}(3)-\mathrm{Zn}(2)-\mathrm{O}(6)$ & $76.84(13)$ \\
\hline $\mathrm{N}(3)-\mathrm{Zn}(2)-\mathrm{O}(7)$ & $135.53(15)$ \\
\hline $\operatorname{Zn}(4)-\operatorname{Zn}(3)-\operatorname{Zn}(2)$ & $59.89(2)$ \\
\hline $\mathrm{O}(3) \# 1-\mathrm{Zn}(3)-\mathrm{Zn}(2)$ & $81.62(7)$ \\
\hline $\mathrm{O}(3) \# 1-\mathrm{Zn}(3)-\mathrm{Zn}(4)$ & $40.09(7)$ \\
\hline $\mathrm{O}(4) \# 1-\mathrm{Zn}(3)-\mathrm{Zn}(2)$ & $107.00(10)$ \\
\hline $\mathrm{O}(4) \# 1-\mathrm{Zn}(3)-\mathrm{Zn}(4)$ & $140.25(11)$ \\
\hline $\mathrm{O}(4) \# 1-\mathrm{Zn}(3)-\mathrm{O}(3) \# 1$ & $170.01(13)$ \\
\hline $\mathrm{O}(4) \# 1-\mathrm{Zn}(3)-\mathrm{O}(6)$ & $110.66(14)$ \\
\hline $\mathrm{O}(4) \# 1-\mathrm{Zn}(3)-\mathrm{O}(7)$ & $104.29(14)$ \\
\hline $\mathrm{O}(4) \# 1-\mathrm{Zn}(3)-\mathrm{N}(2) \# 1$ & $95.41(15)$ \\
\hline $\mathrm{O}(6)-\mathrm{Zn}(3)-\mathrm{Zn}(2)$ & $47.05(8)$ \\
\hline
\end{tabular}




\begin{tabular}{|c|c|}
\hline $\mathrm{O}(6)-\mathrm{Zn}(3)-\mathrm{Zn}(4)$ & $87.75(9)$ \\
\hline $\mathrm{O}(6)-\mathrm{Zn}(3)-\mathrm{O}(3) \# 1$ & $78.82(11)$ \\
\hline $\mathrm{O}(7)-\mathrm{Zn}(3)-\mathrm{Zn}(2)$ & $39.01(9)$ \\
\hline $\mathrm{O}(7)-\mathrm{Zn}(3)-\mathrm{Zn}(4)$ & $40.37(9)$ \\
\hline $\mathrm{O}(7)-\mathrm{Zn}(3)-\mathrm{O}(3) \# 1$ & $79.07(12)$ \\
\hline $\mathrm{O}(7)-\mathrm{Zn}(3)-\mathrm{O}(6)$ & $85.21(12)$ \\
\hline $\mathrm{N}(2) \# 1-\mathrm{Zn}(3)-\mathrm{Zn}(2)$ & $157.49(11)$ \\
\hline $\mathrm{N}(2) \# 1-\mathrm{Zn}(3)-\mathrm{Zn}(4)$ & $101.22(12)$ \\
\hline $\mathrm{N}(2) \# 1-\mathrm{Zn}(3)-\mathrm{O}(3) \# 1$ & $75.87(13)$ \\
\hline $\mathrm{N}(2) \# 1-\mathrm{Zn}(3)-\mathrm{O}(6)$ & $126.48(13)$ \\
\hline $\mathrm{N}(2) \# 1-\mathrm{Zn}(3)-\mathrm{O}(7)$ & $133.39(15)$ \\
\hline $\mathrm{Zn}(3)-\mathrm{Zn}(4)-\mathrm{Zn}(2)$ & $60.64(2)$ \\
\hline $\mathrm{O}(1)-\mathrm{Zn}(4)-\mathrm{Zn}(2)$ & $142.20(12)$ \\
\hline $\mathrm{O}(1)-\mathrm{Zn}(4)-\mathrm{Zn}(3)$ & $105.26(11)$ \\
\hline $\mathrm{O}(1)-\mathrm{Zn}(4)-\mathrm{O}(2)$ & $170.66(13)$ \\
\hline $\mathrm{O}(1)-\mathrm{Zn}(4)-\mathrm{O}(3) \# 1$ & $109.59(14)$ \\
\hline $\mathrm{O}(1)-\mathrm{Zn}(4)-\mathrm{O}(7)$ & $105.87(14)$ \\
\hline $\mathrm{O}(1)-\mathrm{Zn}(4)-\mathrm{N}(1)$ & $94.73(15)$ \\
\hline $\mathrm{O}(2)-\mathrm{Zn}(4)-\mathrm{Zn}(2)$ & $40.31(8)$ \\
\hline $\mathrm{O}(2)-\mathrm{Zn}(4)-\mathrm{Zn}(3)$ & $83.61(8)$ \\
\hline $\mathrm{O}(3) \# 1-\mathrm{Zn}(4)-\mathrm{Zn}(2)$ & $87.32(9)$ \\
\hline $\mathrm{O}(3) \# 1-\mathrm{Zn}(4)-\mathrm{Zn}(3)$ & $48.42(8)$ \\
\hline $\mathrm{O}(3) \# 1-\mathrm{Zn}(4)-\mathrm{O}(2)$ & $78.38(11)$ \\
\hline $\mathrm{O}(3) \# 1-\mathrm{Zn}(4)-\mathrm{O}(7)$ & $86.83(12)$ \\
\hline $\mathrm{O}(7)-\mathrm{Zn}(4)-\mathrm{Zn}(2)$ & $39.62(9)$ \\
\hline $\mathrm{O}(7)-\mathrm{Zn}(4)-\mathrm{Zn}(3)$ & $39.99(9)$ \\
\hline $\mathrm{O}(7)-\mathrm{Zn}(4)-\mathrm{O}(2)$ & $78.93(12)$ \\
\hline $\mathrm{N}(1)-\mathrm{Zn}(4)-\mathrm{Zn}(2)$ & $99.89(12)$ \\
\hline $\mathrm{N}(1)-\mathrm{Zn}(4)-\mathrm{Zn}(3)$ & $159.23(12)$ \\
\hline $\mathrm{N}(1)-\mathrm{Zn}(4)-\mathrm{O}(2)$ & $76.17(14)$ \\
\hline $\mathrm{N}(1)-\mathrm{Zn}(4)-\mathrm{O}(3) \# 1$ & $129.51(15)$ \\
\hline $\mathrm{N}(1)-\mathrm{Zn}(4)-\mathrm{O}(7)$ & $128.89(16)$ \\
\hline $\mathrm{C}(6)-\mathrm{O}(1)-\mathrm{Zn}(4)$ & $126.5(3)$ \\
\hline $\mathrm{Zn}(1)-\mathrm{O}(2)-\mathrm{Zn}(4)$ & $93.72(11)$ \\
\hline $\mathrm{Zn}(2)-\mathrm{O}(2)-\mathrm{Zn}(1)$ & $102.82(14)$ \\
\hline $\mathrm{Zn}(2)-\mathrm{O}(2)-\mathrm{Zn}(4)$ & $91.39(11)$ \\
\hline $\mathrm{C}(17)-\mathrm{O}(2)-\mathrm{Zn}(1)$ & $121.6(2)$ \\
\hline $\mathrm{C}(17)-\mathrm{O}(2)-\mathrm{Zn}(2)$ & $128.8(2)$ \\
\hline $\mathrm{C}(17)-\mathrm{O}(2)-\mathrm{Zn}(4)$ & $108.4(2)$ \\
\hline $\mathrm{Zn}(1)-\mathrm{O}(3)-\mathrm{Zn}(3) \# 1$ & $94.58(11)$ \\
\hline $\mathrm{Zn}(4) \# 1-\mathrm{O}(3)-\mathrm{Zn}(1)$ & $105.52(13)$ \\
\hline $\mathrm{Zn}(4) \# 1-\mathrm{O}(3)-\mathrm{Zn}(3) \# 1$ & $91.49(11)$ \\
\hline $\mathrm{C}(25)-\mathrm{O}(3)-\mathrm{Zn}(1)$ & $122.9(2)$ \\
\hline $\mathrm{C}(25)-\mathrm{O}(3)-\mathrm{Zn}(3) \# 1$ & $110.2(2)$ \\
\hline
\end{tabular}




$\begin{array}{lc}\mathrm{C}(25)-\mathrm{O}(3)-\mathrm{Zn}(4) \# 1 & 123.5(2) \\ \mathrm{C}(34)-\mathrm{O}(4)-\mathrm{Zn}(3) \# 1 & 127.1(3) \\ \mathrm{C}(47)-\mathrm{O}(5)-\mathrm{Zn}(2) & 127.3(3) \\ \mathrm{Zn}(1)-\mathrm{O}(6)-\mathrm{Zn}(2) & 94.02(11) \\ \mathrm{Zn}(3)-\mathrm{O}(6)-\mathrm{Zn}(1) & 102.62(12) \\ \mathrm{Zn}(3)-\mathrm{O}(6)-\mathrm{Zn}(2) & 92.56(11) \\ \mathrm{C}(63)-\mathrm{O}(6)-\mathrm{Zn}(1) & 122.5(2) \\ \mathrm{C}(63)-\mathrm{O}(6)-\mathrm{Zn}(2) & 109.78(18) \\ \mathrm{C}(63)-\mathrm{O}(6)-\mathrm{Zn}(3) & 126.5(2) \\ \mathrm{Zn}(2)-\mathrm{O}(7)-\mathrm{Zn}(3) & 101.91(14) \\ \mathrm{Zn}(2)-\mathrm{O}(7)-\mathrm{Zn}(4) & 100.32(14) \\ \mathrm{Zn}(3)-\mathrm{O}(7)-\mathrm{Zn}(4) & 99.64(14) \\ \mathrm{C}(15)-\mathrm{N}(1)-\operatorname{Zn}(4) & 120.8(3) \\ \mathrm{C}(16)-\mathrm{N}(1)-\operatorname{Zn}(4) & 118.6(3) \\ \mathrm{C}(26)-\mathrm{N}(2)-\operatorname{Zn}(3) \# 1 & 119.5(3) \\ \mathrm{C}(32)-\mathrm{N}(2)-\operatorname{Zn}(3) \# 1 & 120.8(3) \\ \mathrm{C}(61)-\mathrm{N}(3)-\operatorname{Zn}(2) & 120.5(3) \\ \mathrm{C}(62)-\mathrm{N}(3)-\operatorname{Zn}(2) & 118.5(2)\end{array}$

Symmetry transformations used to generate equivalent atoms: $\# 1-\mathrm{x}+2, \mathrm{y},-\mathrm{z}+3 / 2 \quad \# 2-\mathrm{x}+1 / 2,-\mathrm{y}+1 / 2,-\mathrm{z}+2$ 


\section{Additional X-ray crystallographic structures of 1 and 2}

6.1. Figure S1. View of the asymmetric unit of 1

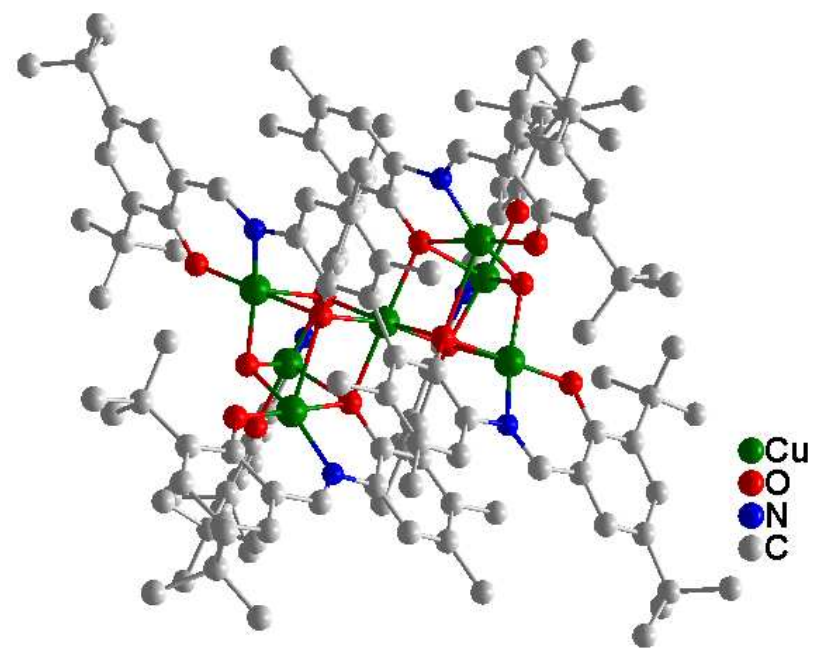

6.2. Figure S2. View of the asymmetric unit of 2

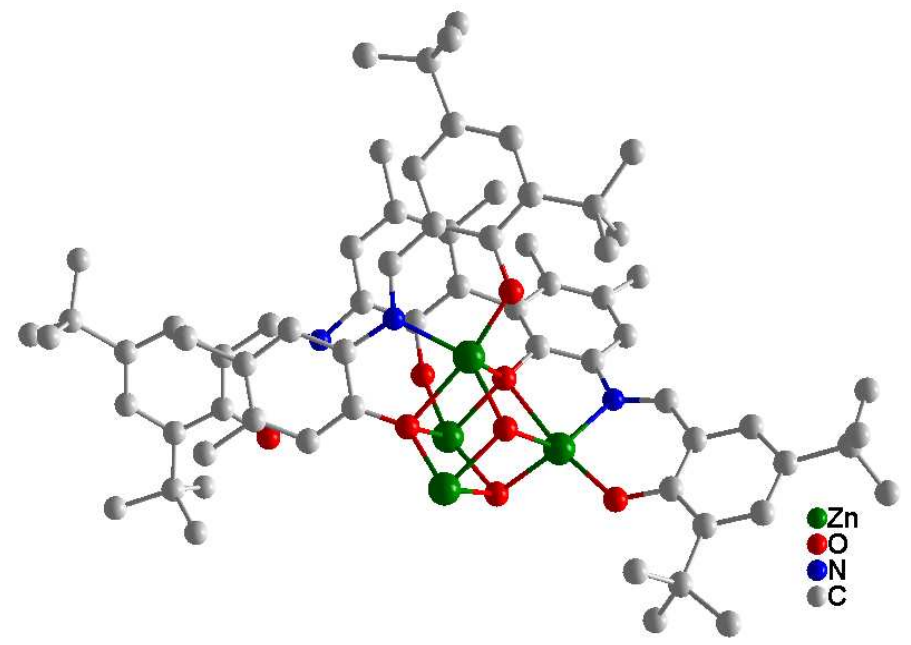

6.3. Figure S3. Coordination environments of the dicubane cluster in $\mathbf{1}$ (a) and $\mathbf{2}$ (b).

(a)

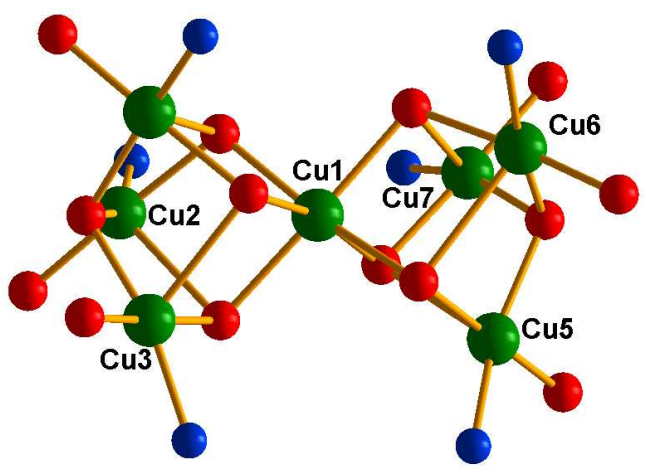

(b)

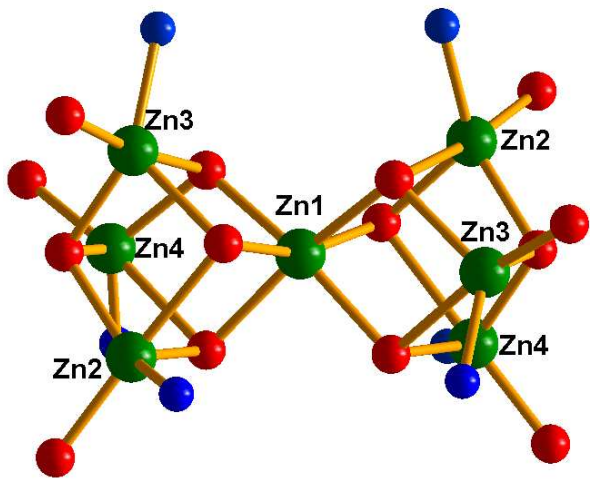




\section{UV/Vis absorption and fluorescent emission spectra}

7.1. Figure S4. UV-Vis Spectra of $\mathbf{L}-(2 \mathrm{MOM}-2 \mathrm{H}), \mathbf{1}, \mathbf{2}$ and the recovered 1 after catalytic reaction (in $\mathrm{CHCl}_{3}$ ).

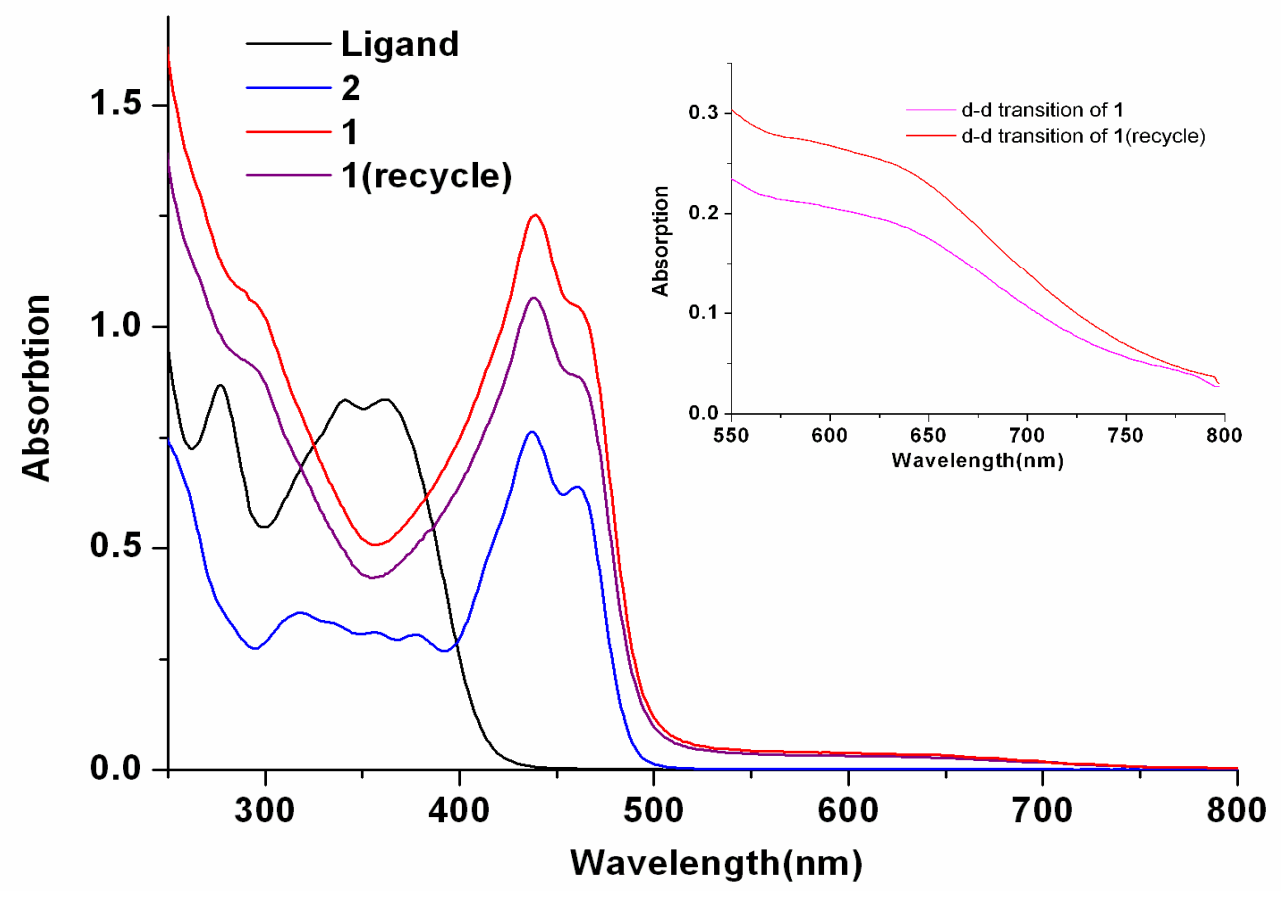

7.2. Figure S5. UV-Vis Spectra of $\mathbf{1}$ in the solid state.

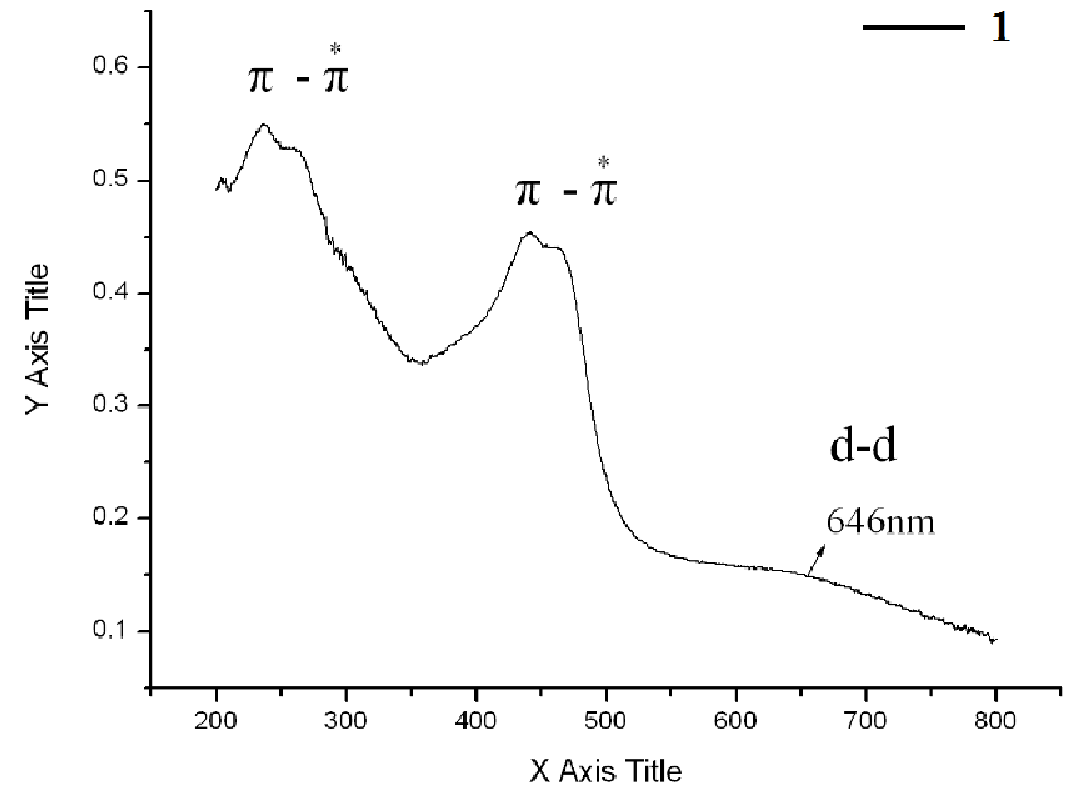


7.3. Figure S6. Fluorescence Spectra of L-(2MOM-2H), 1 and 2.

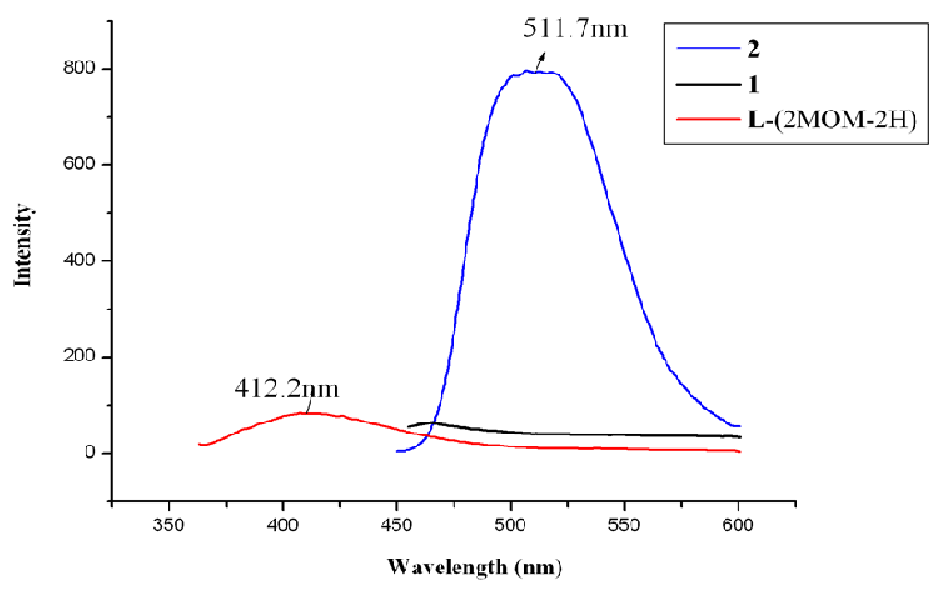

\section{Experimental procedure for Catalytic property of 1}

In a small flask, $1(0.8 \%$ eq, $15.3-22.1 \mathrm{mg})$ and cycloalkanes or benzene series $(0.1 \mathrm{~mL})$ were placed in $3 \mathrm{~mL} \mathrm{CH} \mathrm{CH}_{3} \mathrm{CN}$. After thermal equilibration $\left(55^{\circ} \mathrm{C}\right), \mathrm{H}_{2} \mathrm{O}_{2}$ (3.97-28.76 mmol, 30\% aqueous solution) was added slowly. The reaction mixture was stirred for $4-6 \mathrm{~h}$ and cooled to room temperature. Then $60 \mu \mathrm{L}$ of cyclooctanone (for oxidation of cyclopentanone) or cyclopentanone (for other substrates) as internal standard reagent and $15.00 \mathrm{~mL}$ diethyl ether (to extract the substrate and the products from the reaction mixture) were added. The obtained mixture was stirred during 10 min and then a sample was taken from the organic phase and analyzed by GC using a Lunan Ruihong SP-6890 equipped with a flame ionization detector and silica capillary column SP-56, and SHIMADZU GC-2010 with a coupled GCMS-QP2010, using GCMS Solution Version 2.5(SHIMADZU Corp.)

Blank experiments were performed with different amounts of $\mathrm{H}_{2} \mathrm{O}_{2}$ and other reagents without catalyst, and confirmed that no cycloalkane oxidation products or only trace amount of benzene series oxidation was obtained unless the copper catalyst was used. 
9. Table S4. Peroxidative oxidation of toluene and ethyl benzene ${ }^{[\mathrm{a}]}$

\begin{tabular}{|c|c|c|c|c|c|c|}
\hline $\begin{array}{l}R=1 \\
R=1\end{array}$ & & & & & & \\
\hline \multirow[b]{2}{*}{ Entry } & \multirow[b]{2}{*}{ Substrate } & \multirow[b]{2}{*}{$\mathrm{T}^{\circ} \mathrm{C}$} & \multirow[b]{2}{*}{$\begin{array}{l}\mathrm{n}\left(\mathrm{H}_{2} \mathrm{O}_{2}\right) \\
\mathrm{n} \text { (cat.) }\end{array}$} & \multirow{2}{*}{ Time/h } & \multicolumn{2}{|c|}{ Yield $(\%)$ of product ${ }^{\text {lb }}$} \\
\hline & & & & & Ketone & Alcohol \\
\hline 1 & 3d & $25^{\circ} \mathrm{C}$ & 3360 & 72 & 2.33 & trace \\
\hline 2 & 3d & $55^{\circ} \mathrm{C}$ & 670 & 4 & 7.87 & trace \\
\hline 3 & 3d & $55^{\circ} \mathrm{C}$ & 3360 & 6 & 10.15 & trace \\
\hline 4 & $3 e$ & $25^{\circ} \mathrm{C}$ & 3360 & 72 & 3.91 & trace \\
\hline 5 & $3 e$ & $55^{\circ} \mathrm{C}$ & 670 & 4 & 12.21 & trace \\
\hline 6 & $3 e$ & $55^{\circ} \mathrm{C}$ & 3360 & 6 & 14.11 & trace \\
\hline 7 & 3d & $55^{\circ} \mathrm{C}$ & $N / A^{[c]}$ & 72 & n.d. & n.d. ${ }^{[\mathrm{d}]}$ \\
\hline
\end{tabular}

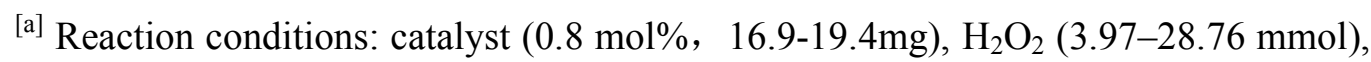
substrates $(0.1 \mathrm{ml}){ }^{[\mathrm{b}]}$ Moles of product/100 moles of substract. ${ }^{[\mathrm{c}]}$ no catalyst but excess $\mathrm{H}_{2} \mathrm{O}_{2}$ was added. ${ }^{[\mathrm{d}]}$ not detected by GC-MS. 


\section{GC and GC-MS results}

(a) GC trace for oxidation of cyclopentane.

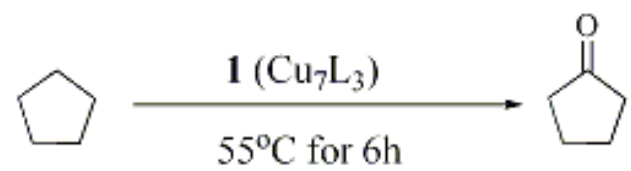

Temperature program: initial time $=0 \mathrm{~min}$, fixed temperature $=55^{\circ} \mathrm{C}$, final time $=15$ $\min$.
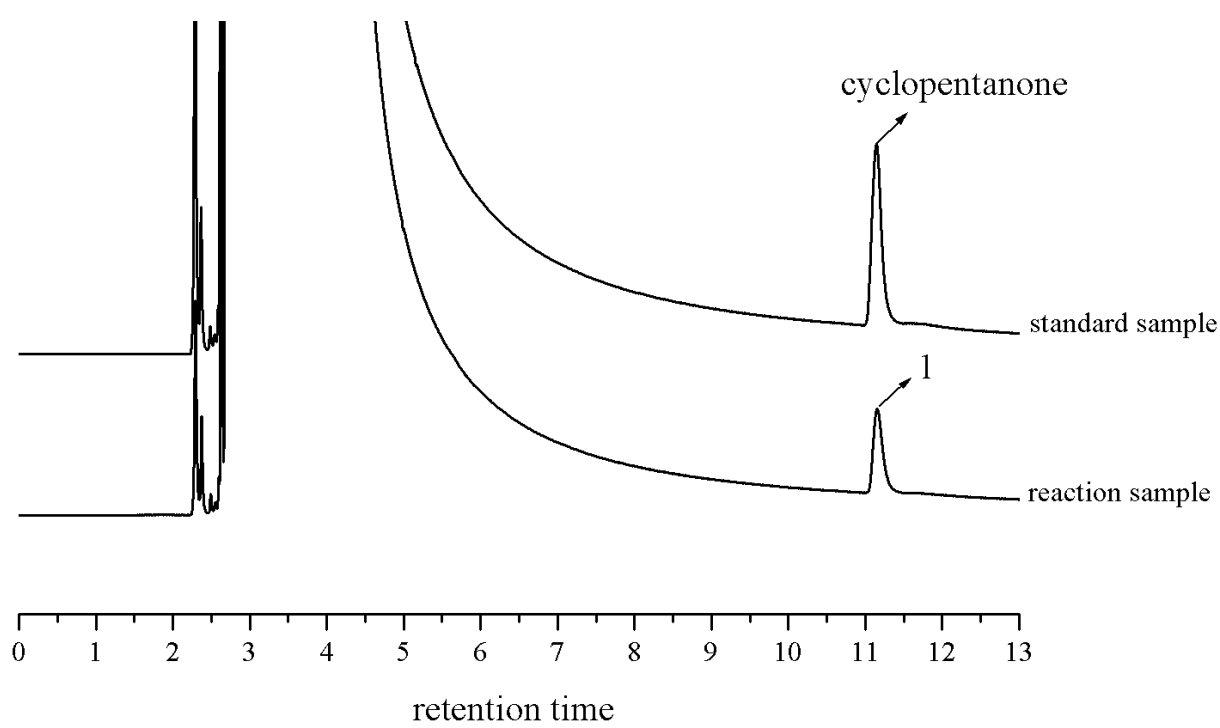

GC-MS spectrum of cyclopentanone $(\mathrm{m} / \mathrm{z}=84)$

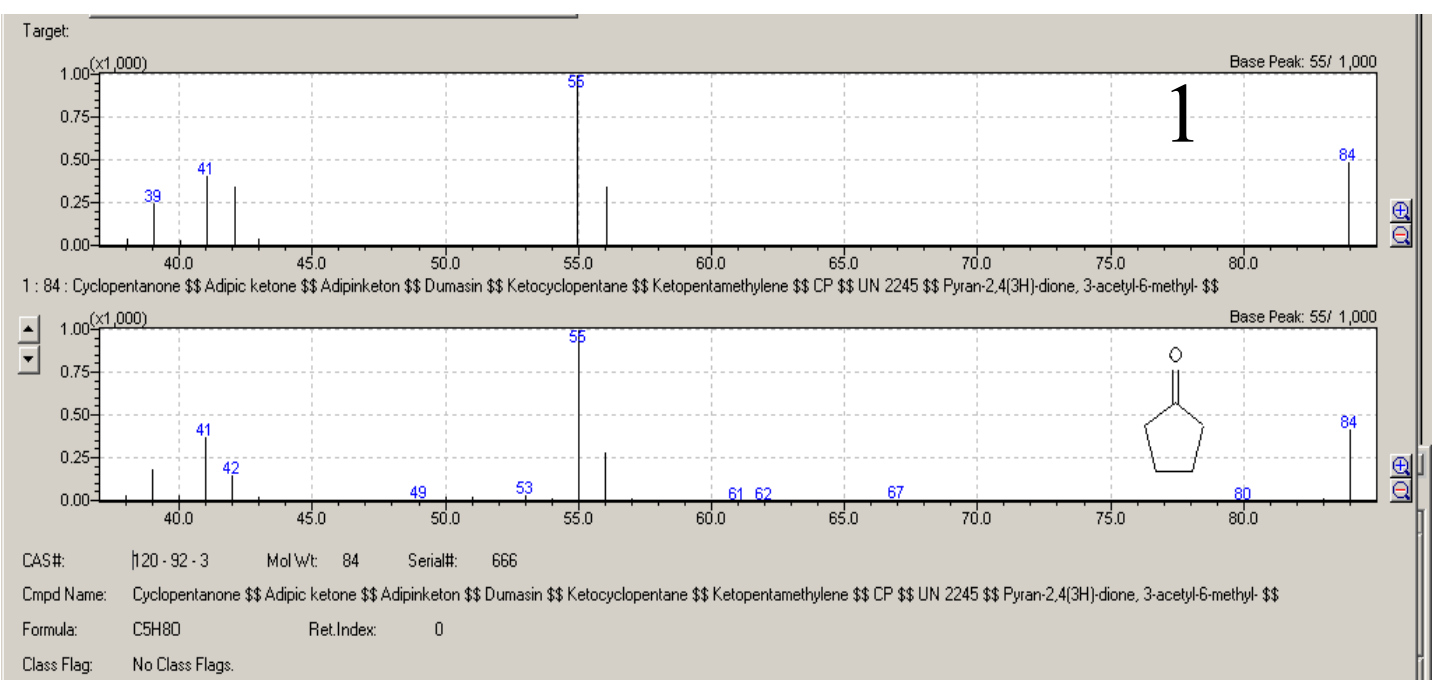


(b) GC trace for oxidation of cyclohexane.

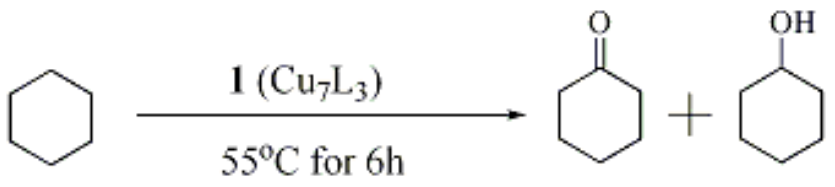

Temperature program: initial time $=0 \mathrm{~min}$, fixed temperature $=55^{\circ} \mathrm{C}$, final time $=25$ min.

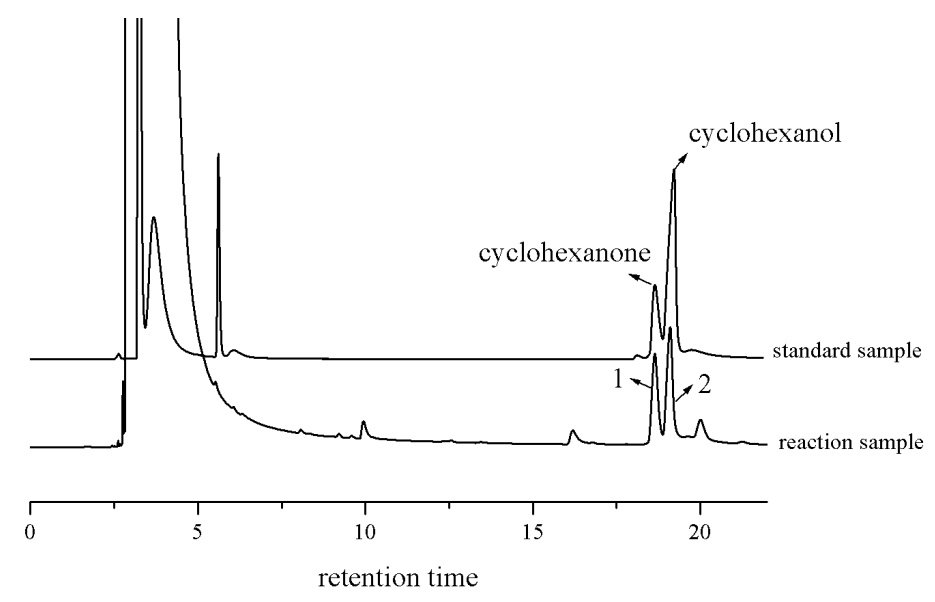

GC-MS spectra of cyclohexanone $(\mathrm{m} / \mathrm{z}=98)$ and cyclohexanol $(\mathrm{m} / \mathrm{z}=100)$

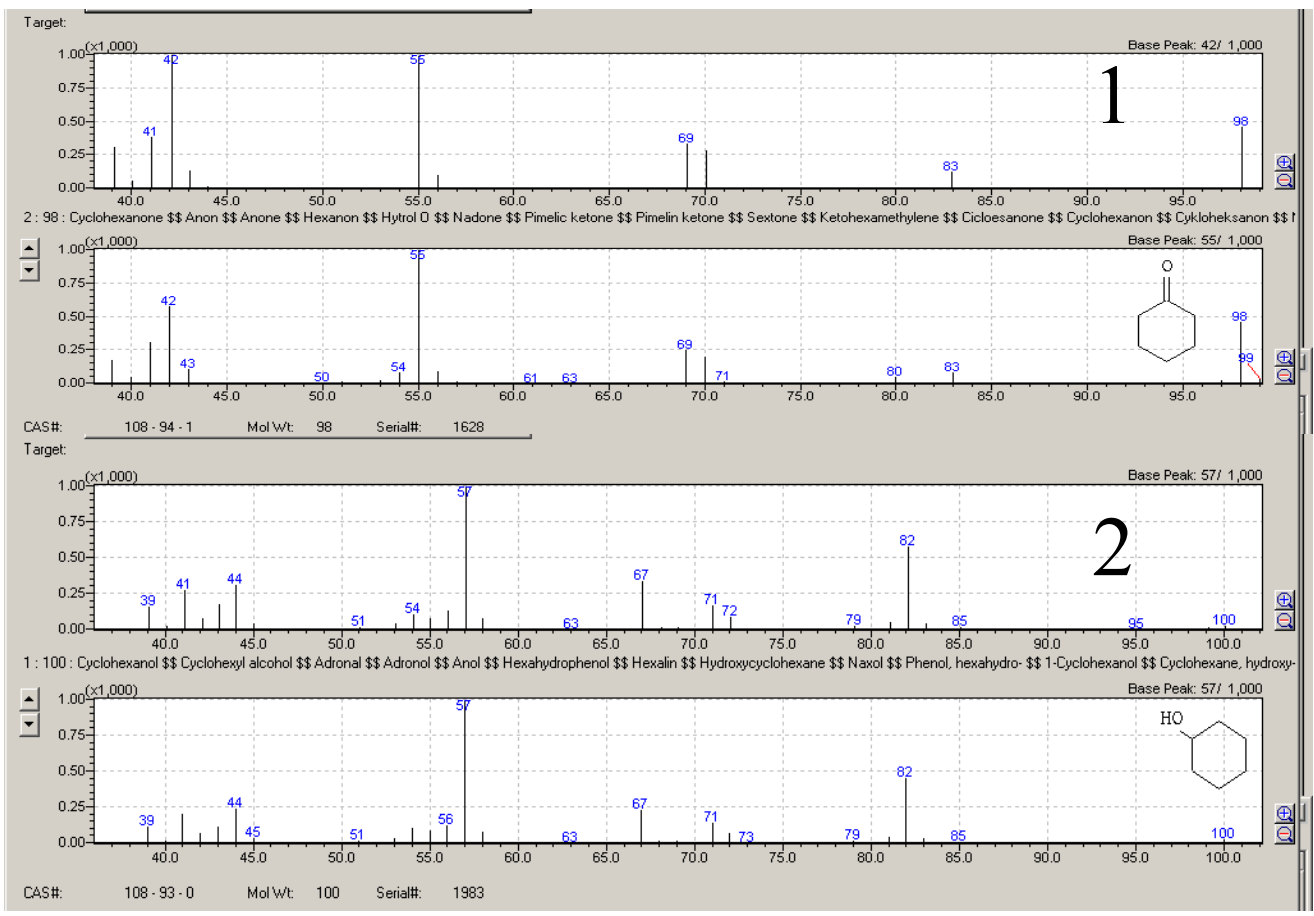


(c) GC trace for oxidation of cyclooctane.

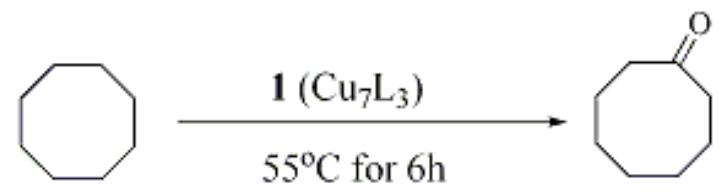

Temperature program: initial time $=0 \mathrm{~min}$, fixed temperature $=85^{\circ} \mathrm{C}$, final time $=55$ $\min$.

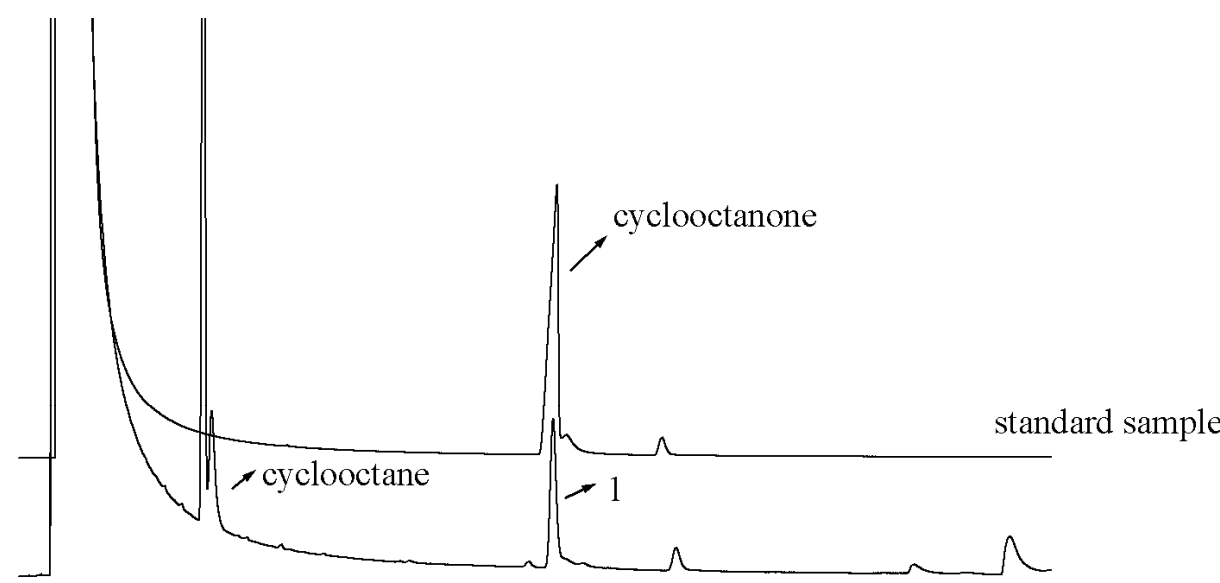

reaction sample

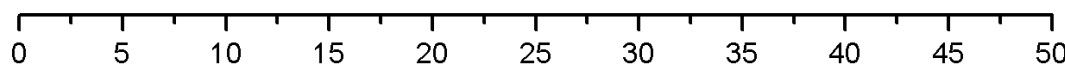

retention time

GC-MS spectrum of cyclooctanone $(\mathrm{m} / \mathrm{z}=126)$.

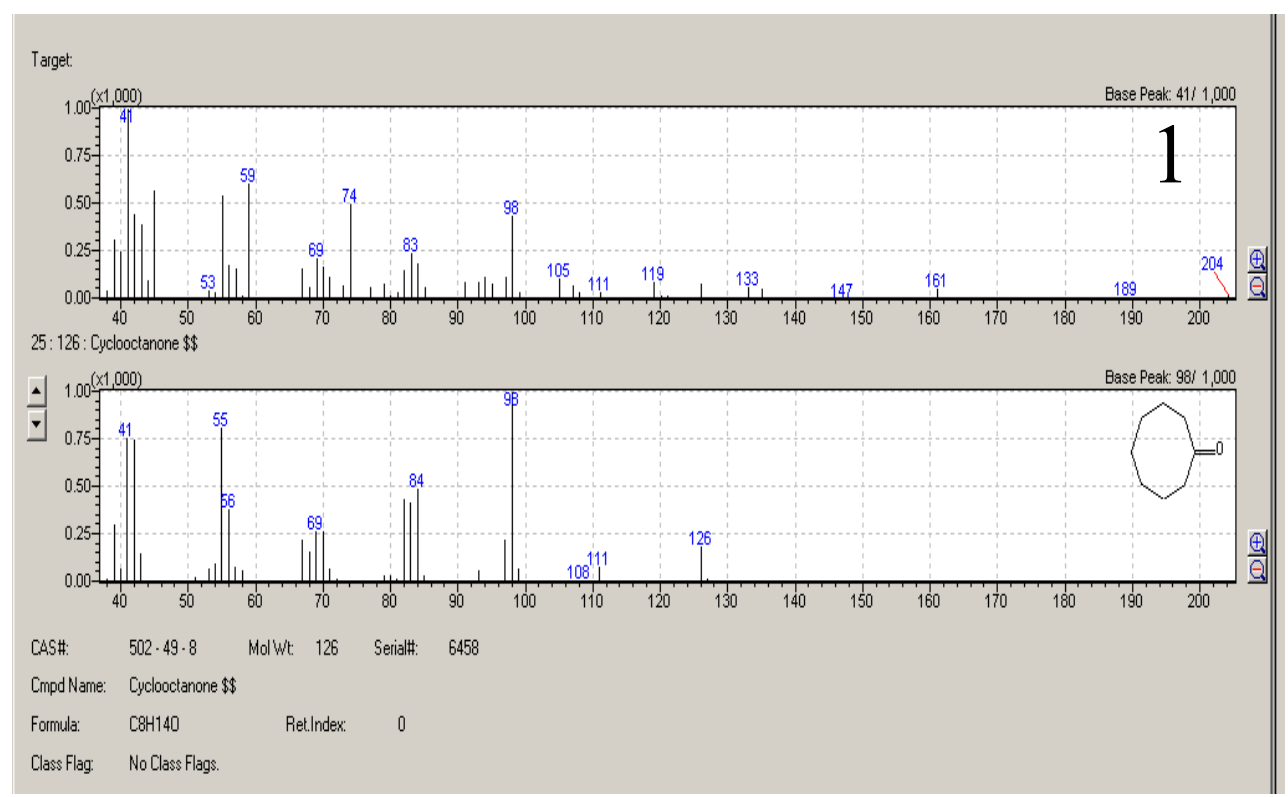


(d) GC trace for oxidation of toluene.

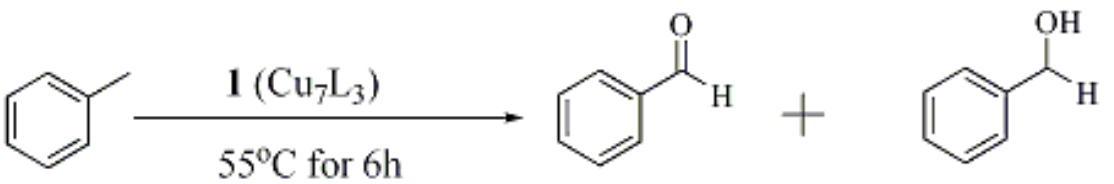

Temperature program: initial time $=0 \mathrm{~min}$, fixed temperature $=85^{\circ} \mathrm{C}$, final time $=27$ min.
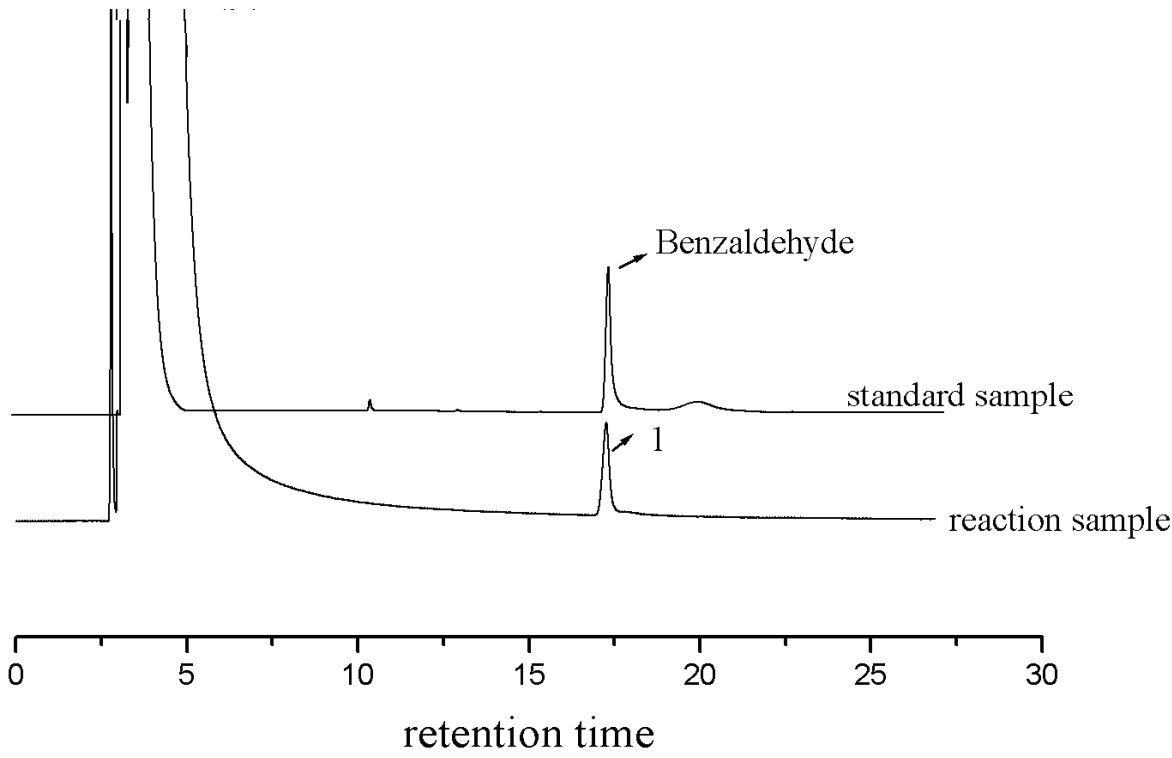

GC-MS spectrum of benzaldehyde $(\mathrm{m} / \mathrm{z}=106)$ and benzyl alcohol $(\mathrm{m} / \mathrm{z}=108)$.

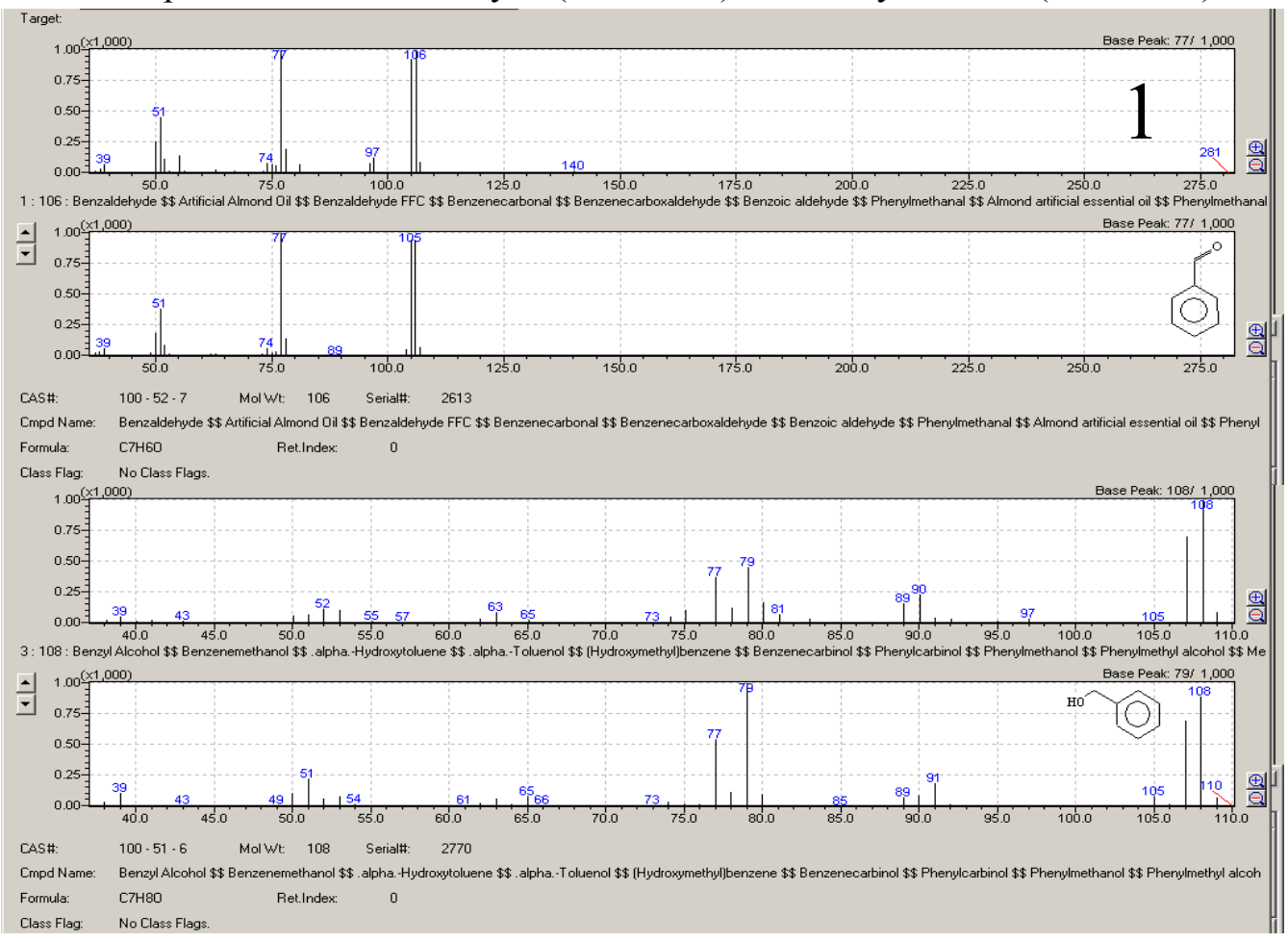


(e) GC trace for oxidation of ethyl benzene.<smiles>CCc1ccccc1</smiles>

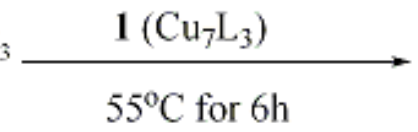<smiles>CC(=O)c1ccccc1</smiles><smiles>CC(O)c1ccccc1</smiles>

Temperature program: initial time $=0 \mathrm{~min}$, fixed temperature $=85^{\circ} \mathrm{C}$, final time $=35$ min.
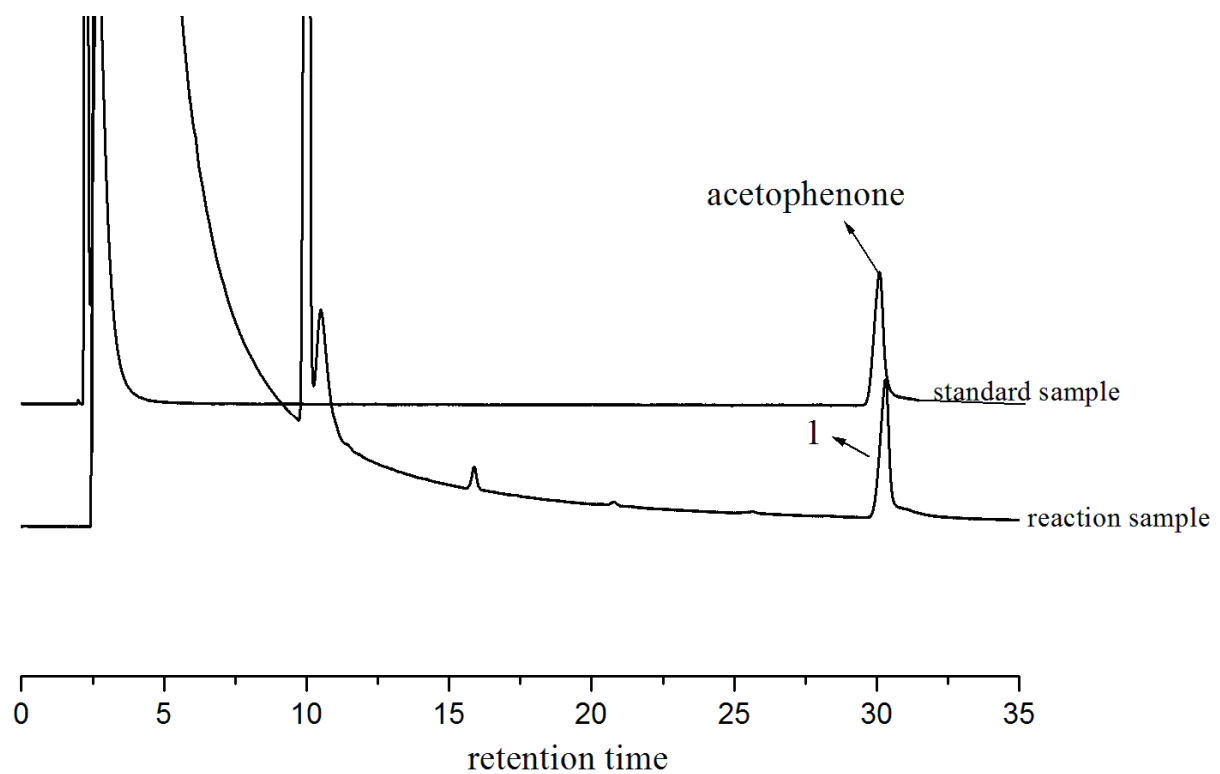

GC-MS spectrum of acetophenone $(\mathrm{m} / \mathrm{z}=120)$ and phenethanol $(\mathrm{m} / \mathrm{z}=122)$.

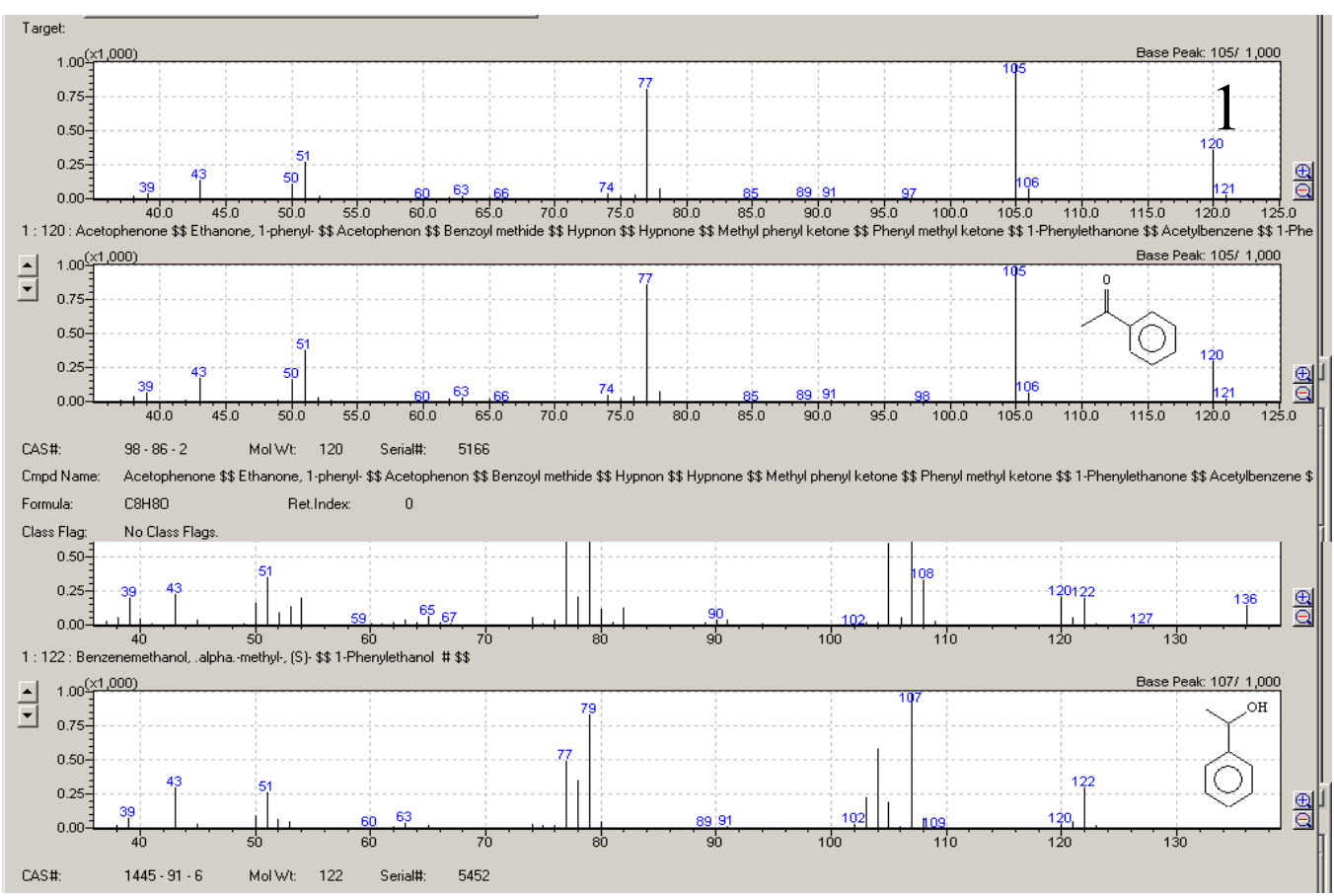




\section{GC trace for oxidation of cyclohexane using recovered catalyst 1}

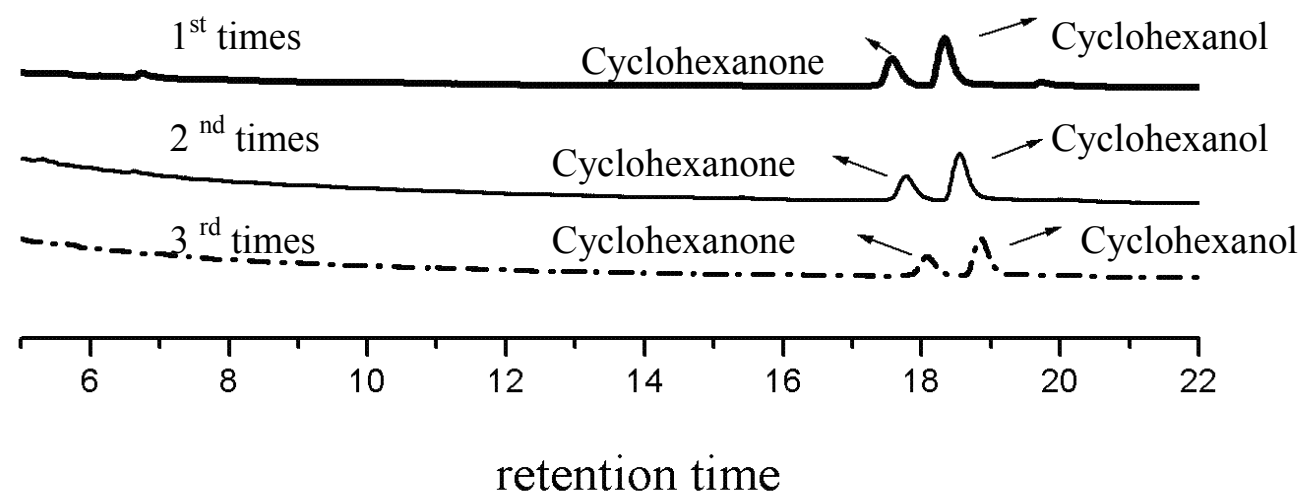

\section{Peroxidative oxidation of cyclohexane in the presence of radical trap}

In a small flask, $1(0.8 \%$ eq, $7.44 \mu \mathrm{mol})$ and cyclohexane $(0.1 \mathrm{ml}, 0.93 \mathrm{mmol})$ was placed in $3 \mathrm{ml} \mathrm{CH}{ }_{3} \mathrm{CN}$. After thermal equilibration $\left(55^{\circ} \mathrm{C}\right), \mathrm{H}_{2} \mathrm{O}_{2}(4.97 \mathrm{mmol}, 30 \%$ aqueous solution) was added by syringe pump. Then carbon-radical trap such as $\mathrm{CBrCl}_{3}$ or TEMPO (2,2,6,6-tetramethylpiperidin-1-oxyl) was added, and also have a blank sample as comparison. After being stirred for $6 \mathrm{~h}$, the cloudy mixture was filtered and the solvent was analyzed by using GC-MS.

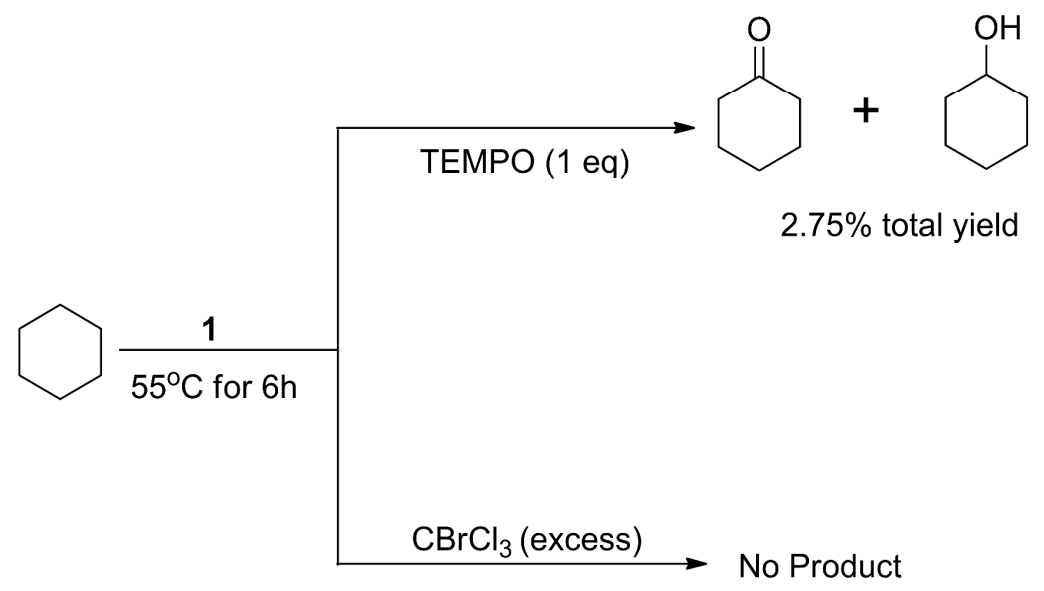

Yield: $\mathrm{CBrCl}_{3}$ almost 0\%; TEMPO 2.75\%; $125.1 \%$.

GC trace for oxidation of cyclohexane. Temperature program: initial time $=0 \mathrm{~min}$, fixed temperature $=55^{\circ} \mathrm{C}$, final time $=25 \mathrm{~min}$. 


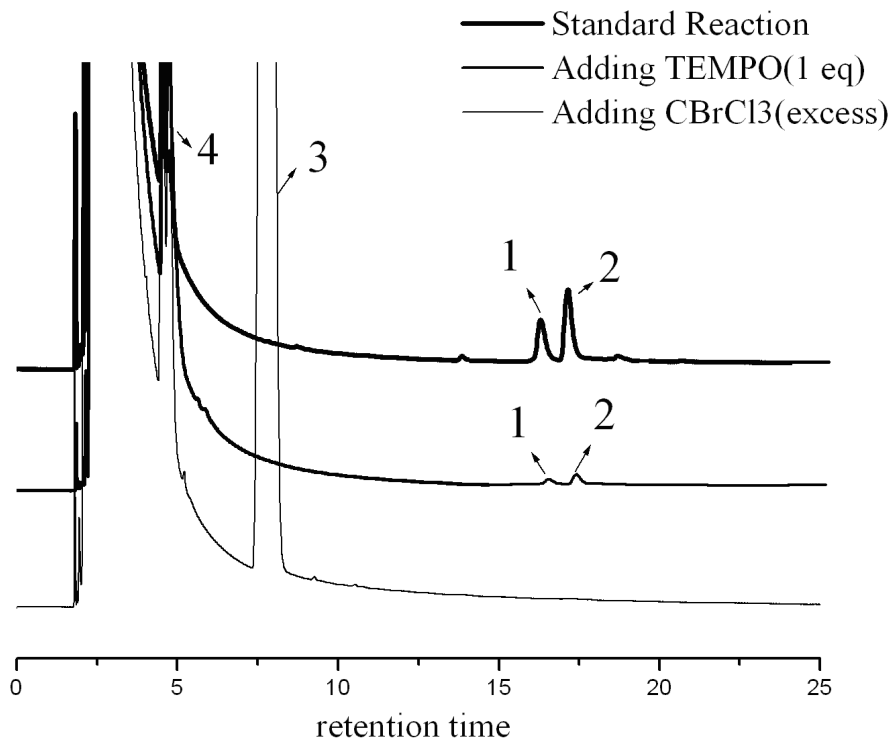

1-cyclohexanone; 2 - cyclohexanol; $3-\mathrm{CBrCl}_{3} ; 4-$ cyclohexane:. 
13. ${ }^{1} \mathrm{H}$ and ${ }^{13} \mathrm{C}$ NMR spectra

13.1. ${ }^{1} \mathrm{H}$ NMR of 2

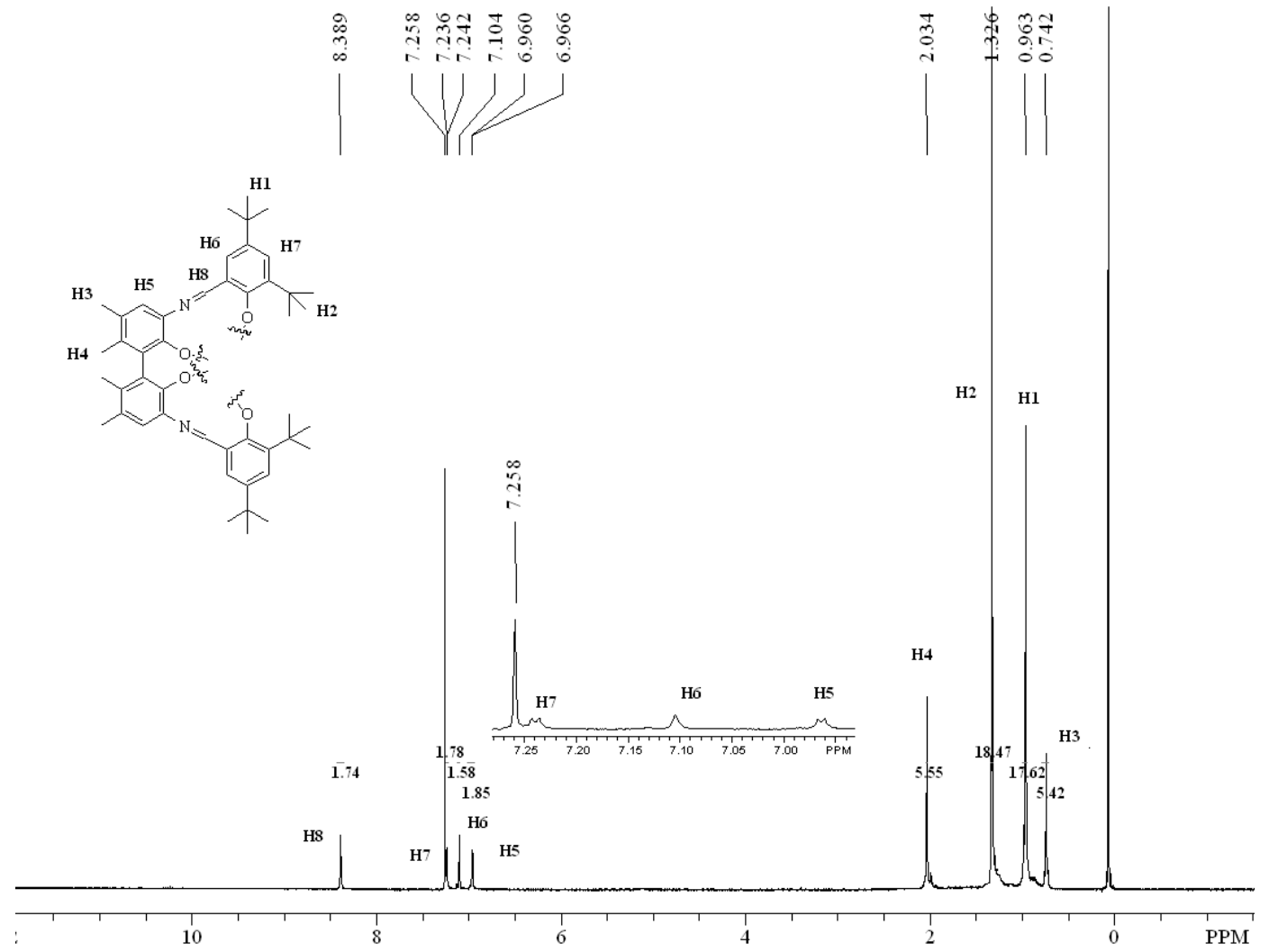

13.2. ${ }^{13} \mathrm{C}$ NMR of 2
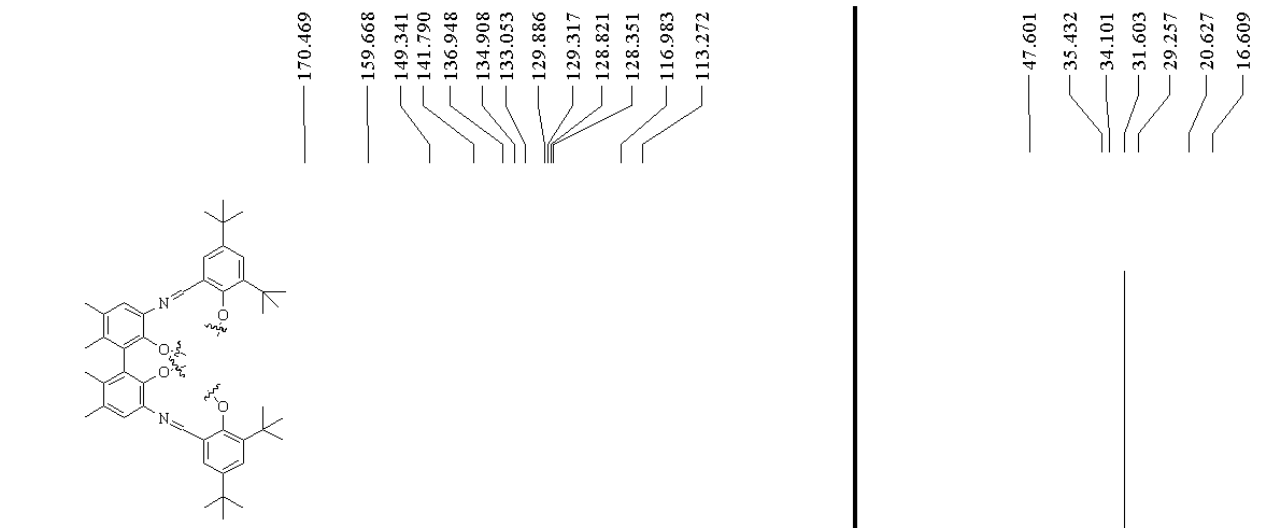
13.3. ${ }^{1} \mathrm{H}$ NMR of the ligand

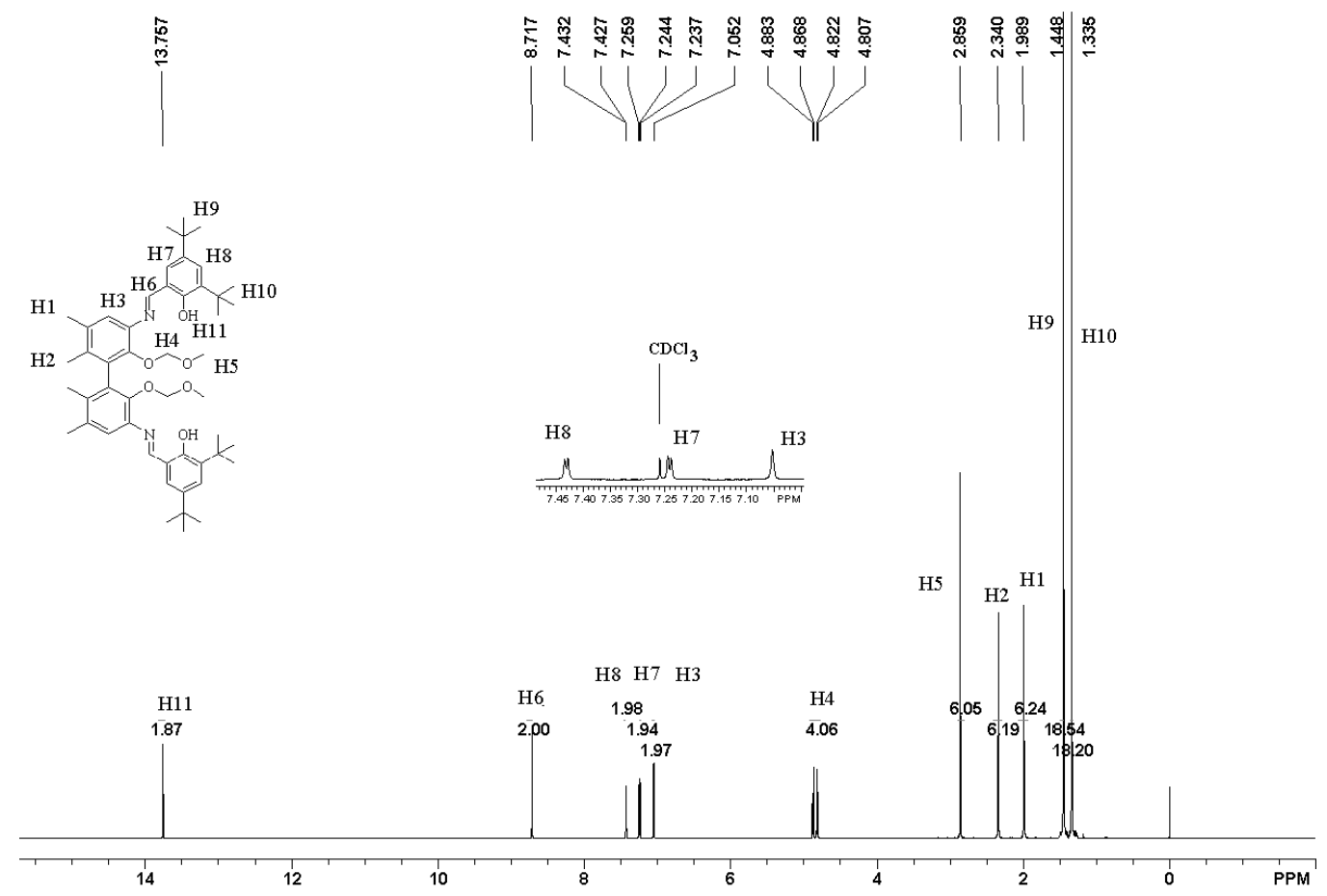

13.4. ${ }^{13} \mathrm{C}$ NMR of the ligand

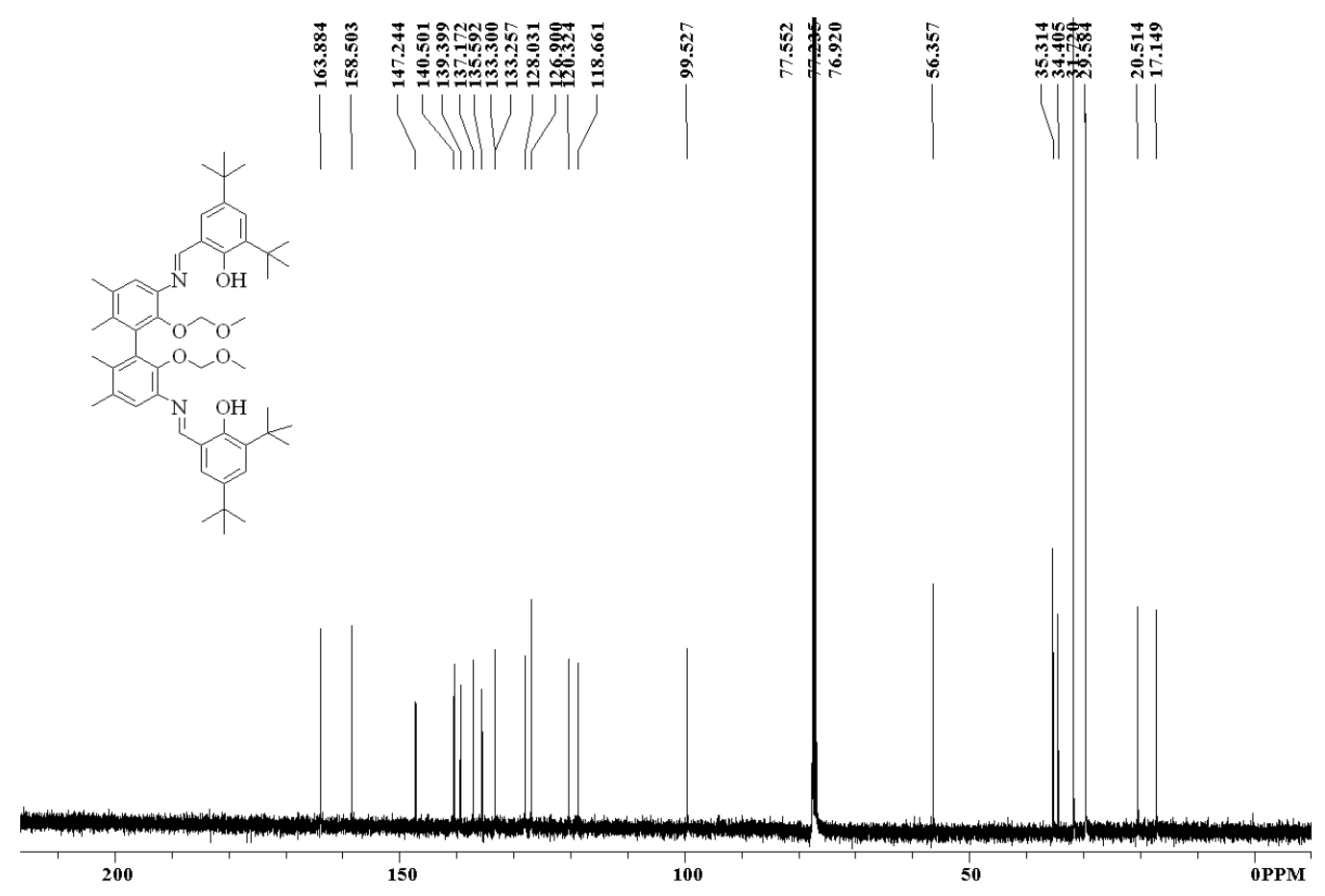




\section{ESI-MS of the reported compounds}

14.1. MS of the ligand $\mathrm{L}-(2 \mathrm{MOM}-2 \mathrm{H})$.

F2000A

Micromass LCT

Analysis\&Research Center ECUST

L81404 212 (3.551) Con (212-194)

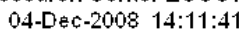

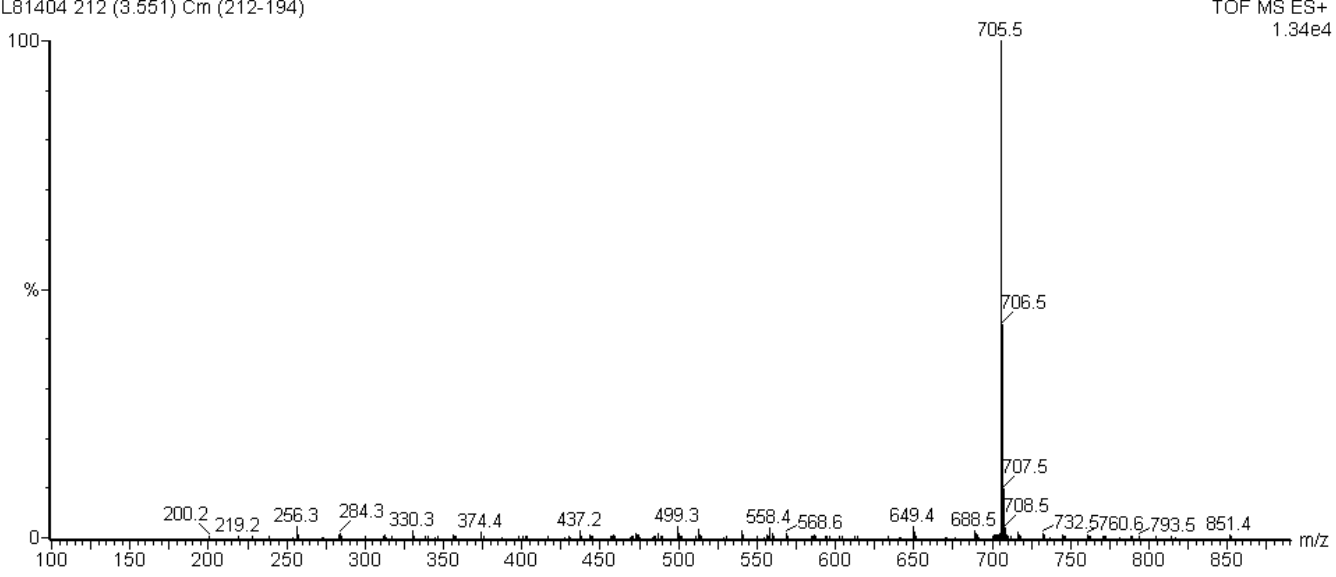

\subsection{MS of 1}

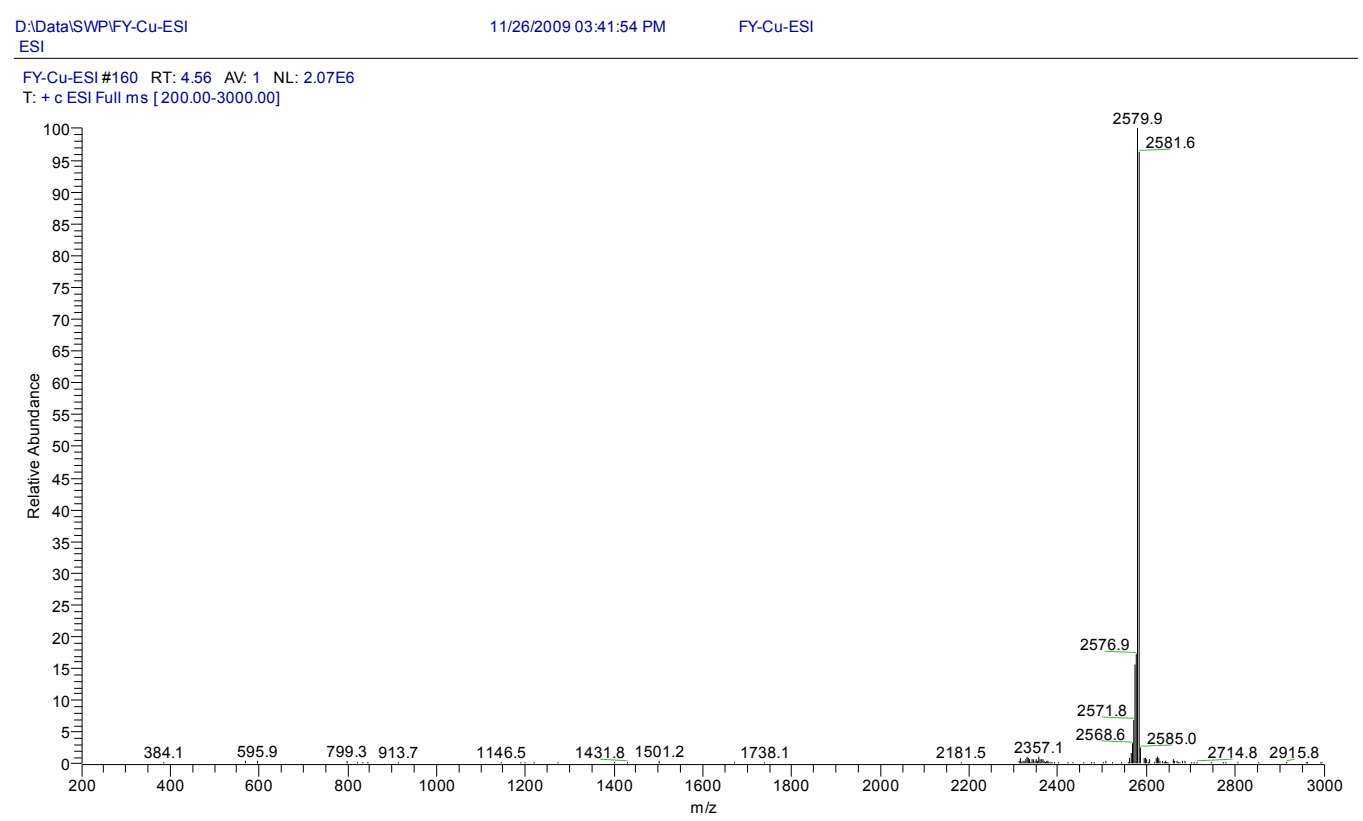




\subsection{MS of 2}

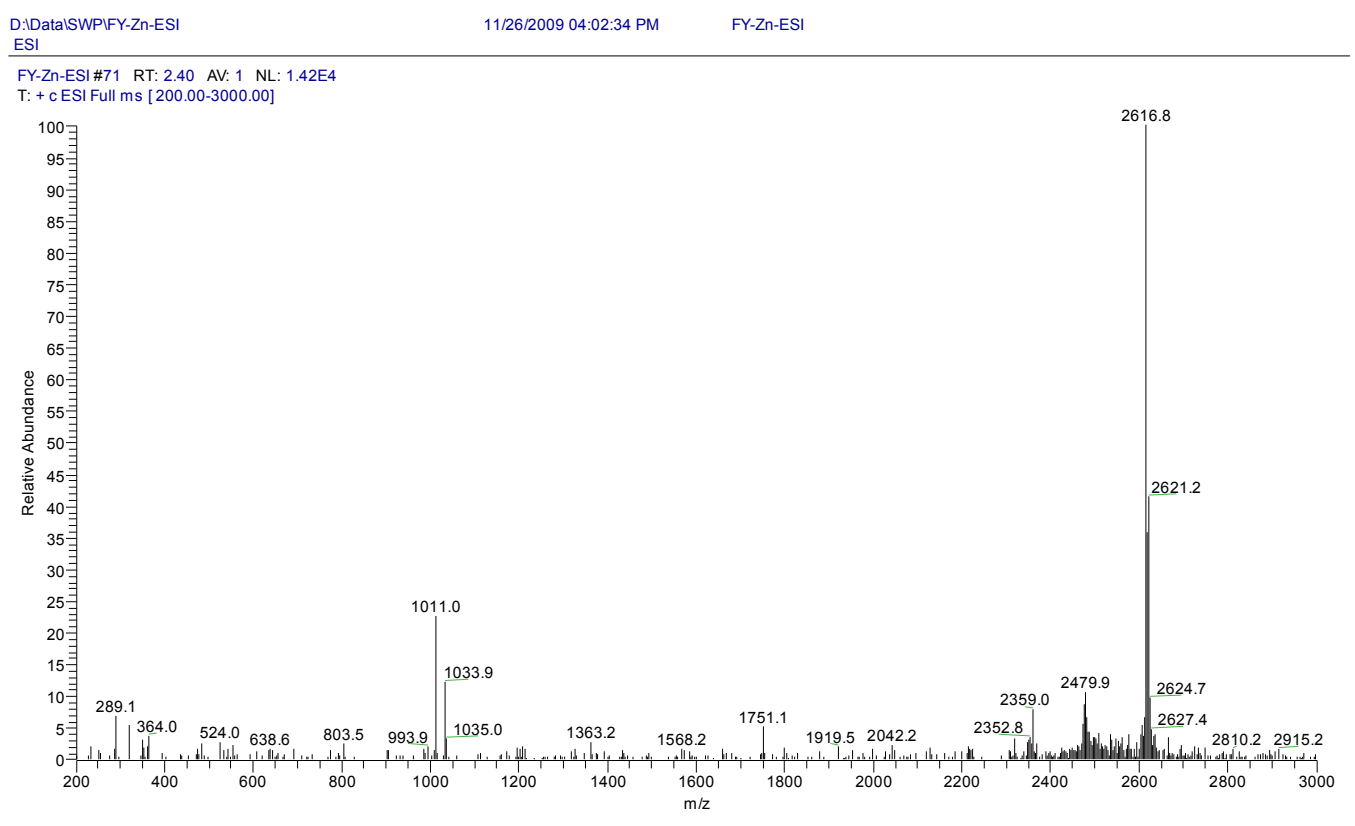

\subsection{ESI- Mass of the recovered 1}

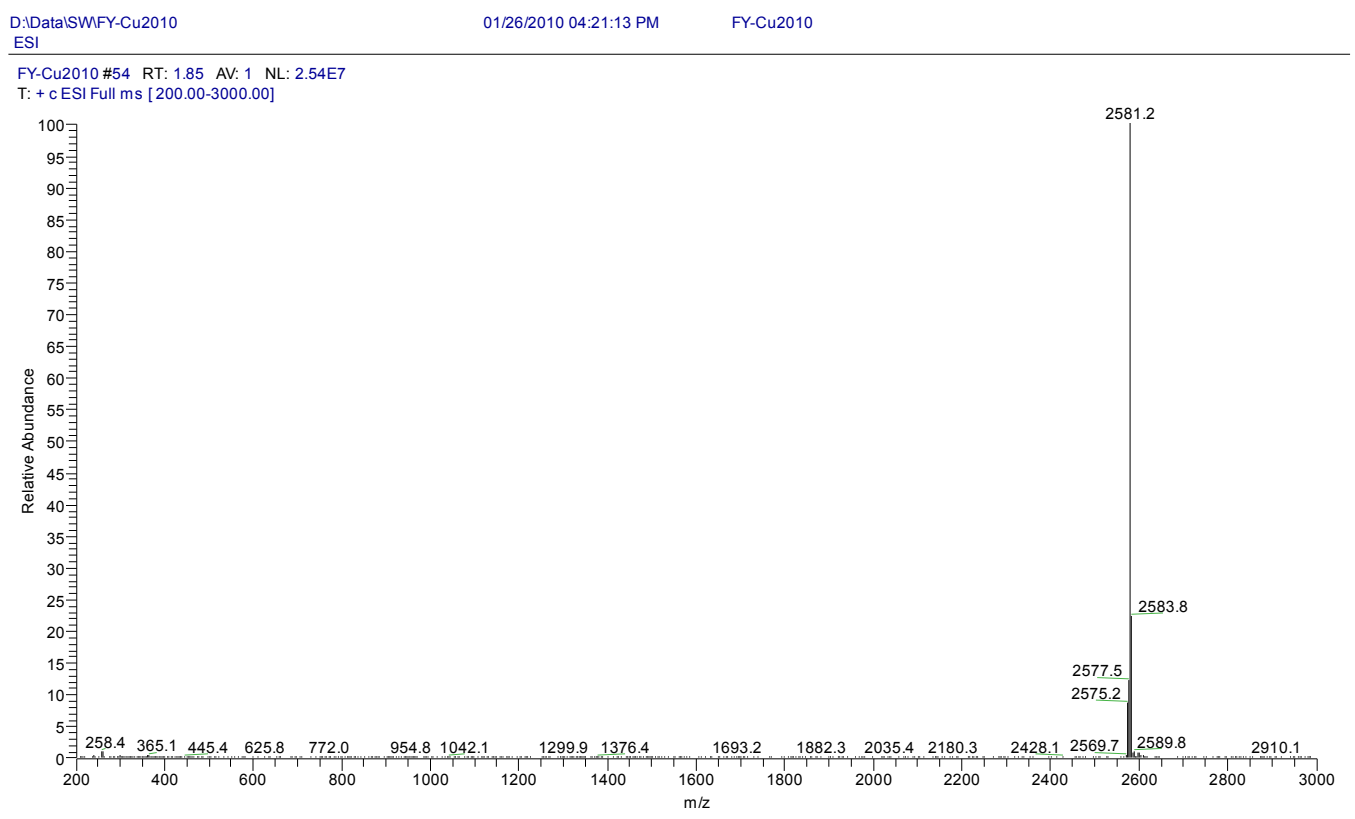

